\title{
Directed energy planetary defense
}

Philip Lubin, Gary Hughes, J. Bible, Jesse Bublitz, Josh Arriola, et al.

Philip Lubin, Gary B. Hughes, J. J. Bible, Jesse Bublitz, Josh Arriola, Caio Motta, Jon Suen, Isabella Johansson, Jordan Riley, Nilou Sarvian, Deborah Clayton-Warwick, Jane Wu, Andrew Milich, Mitch Oleson, Mark Pryor, Peter Krogen, Miikka Kangas, "Directed energy planetary defense," Proc. SPIE 8876, Nanophotonics and Macrophotonics for Space Environments VII, 887602 (24 September 2013); doi: 10.1117/12.2030228

SPIE Event: SPIE Optical Engineering + Applications, 2013, San Diego, California, United States 


\title{
Directed Energy Planetary Defense
}

\author{
Philip Lubin ${ }^{\mathrm{a}}$,Gary B. Hughes ${ }^{\mathrm{b}}$, J. J. Bible ${ }^{\mathrm{a}}$, Jesse Bublitz ${ }^{\mathrm{a}}$, Josh Arriola ${ }^{\mathrm{a}}$, \\ CaioMotta $^{\mathrm{a}}$, Jon Suen ${ }^{\mathrm{a}}$, Isabella Johansson ${ }^{\mathrm{a}}$, Jordan Riley ${ }^{\mathrm{a}}$, NilouSarvian ${ }^{\mathrm{a}}$, Deborah \\ Clayton-Warwick ${ }^{\mathrm{a}}$,Jane $\mathrm{Wu}^{\mathrm{a}}$, Andrew Milich ${ }^{\mathrm{a}}$, Mitch Oleson ${ }^{\mathrm{a}}$, Mark Pryor ${ }^{\mathrm{c}}$, Peter \\ Krogen $^{\text {d }}$ and Miikka Kangas ${ }^{\text {a }}$

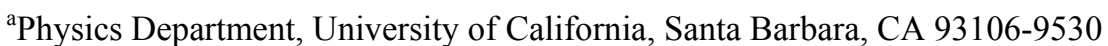 \\ ${ }^{\mathrm{b}}$ Statistics Department, California Polytechnic State University, San Luis Obispo, CA 93407-0405 \\ ${ }^{\mathrm{c}}$ VorticyInc, San Diego, CA 92121 \\ ${ }^{\mathrm{d}}$ Electrical Engineering and Computer Science, MIT,Cambridge, MA02139-4307 \\ lubin@deepspace.ucsb.edu
}

\begin{abstract}
Asteroids and comets that cross Earth's orbit pose a credible risk of impact, with potentially severe disturbances to Earth and society. Numerous risk mitigation strategies have been described, most involving dedicated missions to a threatening object. We propose an orbital planetary defense system capable of heating the surface of potentially hazardous objects to the vaporization point as a feasible approach to impact risk mitigation. We call the system DE-STAR for Directed Energy System for Targeting of Asteroids and exploRation. DE-STAR is a modular phased array of kilowatt class lasers powered by photovoltaic's. Modular design allows for incremental development, test, and initial deployment, lowering cost, minimizing risk, and allowing for technological co-development, leading eventually to an orbiting structure that would be developed in stages with both technological and target milestones. The main objective of DE-STAR is to use the focused directed energy to raise the surface spot temperature to $\sim 3,000 \mathrm{~K}$, allowing direct vaporization of all known substances. In the process of heating the surface ejecting evaporated material a large reaction force would alter the asteroid's orbit. The baseline system is a DE-STAR 3 or 4 (1-10km array) depending on the degree of protection desired. A DE-STAR 4 allows for asteroid engagement starting beyond $1 \mathrm{AU}$ with a spot temperature sufficient to completely evaporate up to 500-m diameter asteroids in one year. Small asteroids and comets can be diverted/evaporated with a DESTAR $2(100 \mathrm{~m})$ while space debris is vaporized with a DE-STAR $1(10 \mathrm{~m})$.
\end{abstract}

Keywords: Asteroid Impact, Directed Energy, Laser Phased Array, Planetary Defense

\section{INTRODUCTION}

Recent advances in photonics make a scientific discussion of directed energy planetary defense feasible whereas even 10 years ago it was close to science fiction. High power lasers are capable of delivering sufficient energy density on a target to melt and vaporize any known material. Laser machining and welding are commonplace in industry, where even refractory metals are directly machined or joined with lasers. Scaling of laser technology has spurred development of directed energy systems that are capable of delivering high energy density on distant targets. Recent developments have resulted in conversion of electrical to photon efficiencies of close to $50 \%$ with powers in excess of one kilowatt per (handheld) unit. Additionally, and critical for this program, such devices can be phased locked. This field is rapidly changing and even more efficient devices with higher power density will be available in the near future. This allows us to contemplate directed energy systems for large scale deployment. Inside the Earth's atmosphere, directed energy systems are hindered by atmospheric fluctuations of the coherent beam. A directed energy system deployed above the atmosphere could project a beam through space unfettered by atmospheric interference and thus allows us to design systems that are essentially diffraction limited as the interplanetary medium (IPM) is extremely tenuous and does not affect the laser beam significantly. This paper describes a feasible design for a future orbiting standoff directed energy system, which we call DE-STAR for Directed Energy System forTargeting of Asteroids and exploRation. The system consists of an array of phase-locked modest power laser amplifiers. By controlling the relative phases of individual laser elements, the combined beam can be directed to a distant target. Lasers are powered by solar photovoltaics of essentially the same area as the laser array. By increasing the array size we can both reduce the spot size due to diffraction and increase the power. This dual effect allows us to vaporizing elements on the surface of asteroids at distances that are 
significant compared to the solar system. By raising the flux $\left(\mathrm{W} / \mathrm{m}^{2}\right)$ on the target asteroid to a sufficiently high level we can begin direct evaporation of the asteroid at the spot. This has two basic effects. Firstly, we directly begin to evaporate the asteroid and given sufficient time, a threatening asteroid could be totally vaporized before hitting the Earth. Secondly, evaporation at the spot causes a back reaction on the asteroid from the vaporization plume which acts as a rocket and thus the asteroid can be deflected. This paper explores the potential capabilities of the system for mitigating the threat of asteroid impact. Since DE-STAR is a phased array consisting of a very large number of elements it can simultaneously be used for multiple purposes and is intrinsically a multi-tasking system. Figure 1 depicts an orbiting DESTAR system simultaneously engaged in both evaporating and deflecting a large asteroid as well as powering and propelling a spacecraft. As this is a modular system we classify each DE-STAR by the log of its linear size, thus a DESTAR 1 is 10 meters, DE-STAR 2 is 100 meters etc. A DE-STAR 4 system will produce a reaction thrust comparable to the Shuttle SRB on the asteroid due to mass ejection and thus allow for orbital diversion of even larger asteroids, beyond several $\mathrm{km}$ in diameter, thus allowing for protection from every known asteroid threat. Smaller systems are also extremely useful. For example, a DE-STAR 2 (100-m size array) would be capable of diverting volatile-laden objects $100 \mathrm{~m}$ in diameter by initiating engagement at $\sim 0.01-0.5 \mathrm{AU}$ ( $\mathrm{AU}=$ Astronomical Unit $=$ mean distance from Earth to Sun $\sim 1.5 \times 10^{11} \mathrm{~m}$ ). Smaller objects could be diverted on shorter notice. The phased array configuration is capable of creating multiple beams, so a single DE-STAR of sufficient size could engage several threats simultaneously, such as a Shoemaker-Levy 9 scenario on Earth. An orbiting DE-STAR would also be capable a wide variety of other functions. Narrow bandwidth and precision beam control would aid narrow search and ephemeris refinement of objects identified with wide-field surveys. Propulsion of kinetic or nuclear tipped asteroid interceptors or other interplanetary spacecraft is possible using the "photon rail gun" mode from direct photon pressure on a spacecraft, propelling a 100-kg craft to $1 \mathrm{AU}$ in 3 days and a 10,000-kg craft to $1 \mathrm{AU}$ in 30 days. A DE-STAR could also provide power to ion propulsion systems, providing both a means of acceleration on the outbound leg, and deceleration for orbit. Ideally two systems would provide the ability to "ping pong" spacecraft if this were needed, though this is vastly more challenging. Vaporization and de-orbiting of debris in Earth orbit could be accomplished with a DE-STAR 1 or 2 system. DE-STAR 3 and 4 arrays may allow standoff interrogation of asteroid composition by observing absorption lines in the blackbody spectrum of a vaporizing surface spot. There are a number of other applications as well, including downlink power via mm, microwave or laser - the so called Space Power System or SPS mode. The system is a standoff planetary defense system that is always ready when needed and no dedicated mission is needed for each threat as is the case with other proposed mitigation methods.

The multi-purpose aspect of the system allows it to be useful with very high "duty cycle". The DE-STAR system is inherently modular and scalable thus allowing us to build and test smaller units both in the lab, on the ground and in sub orbital test flights on balloons. Each module is modest in size and power and identical allowing for mass production.This is key to cost reduction. Each element uses only modest laser power and thus the areal power density is low $\left(<1 \mathrm{~kW} / \mathrm{m}^{2}\right)$. It is inherently redundant since each module is largely self contained and thus failure of modest numbers of elements has little effect. The flux on target $\left(\mathrm{W} / \mathrm{m}^{2}\right)$ at a fixed distance scales as the $\mathrm{d}^{4}$ where $\mathrm{d}$ is the linear dimension of the array and thus it increases very rapidly with increased size. This system is useful for many other purposes, which are briefly mentioned in this paper (and discussed in greater detail in other SPIE Optics \& Photonics 2013 Proceedings papers, including [Hughes et al., 2013] and [Bible et al., 2013]). 

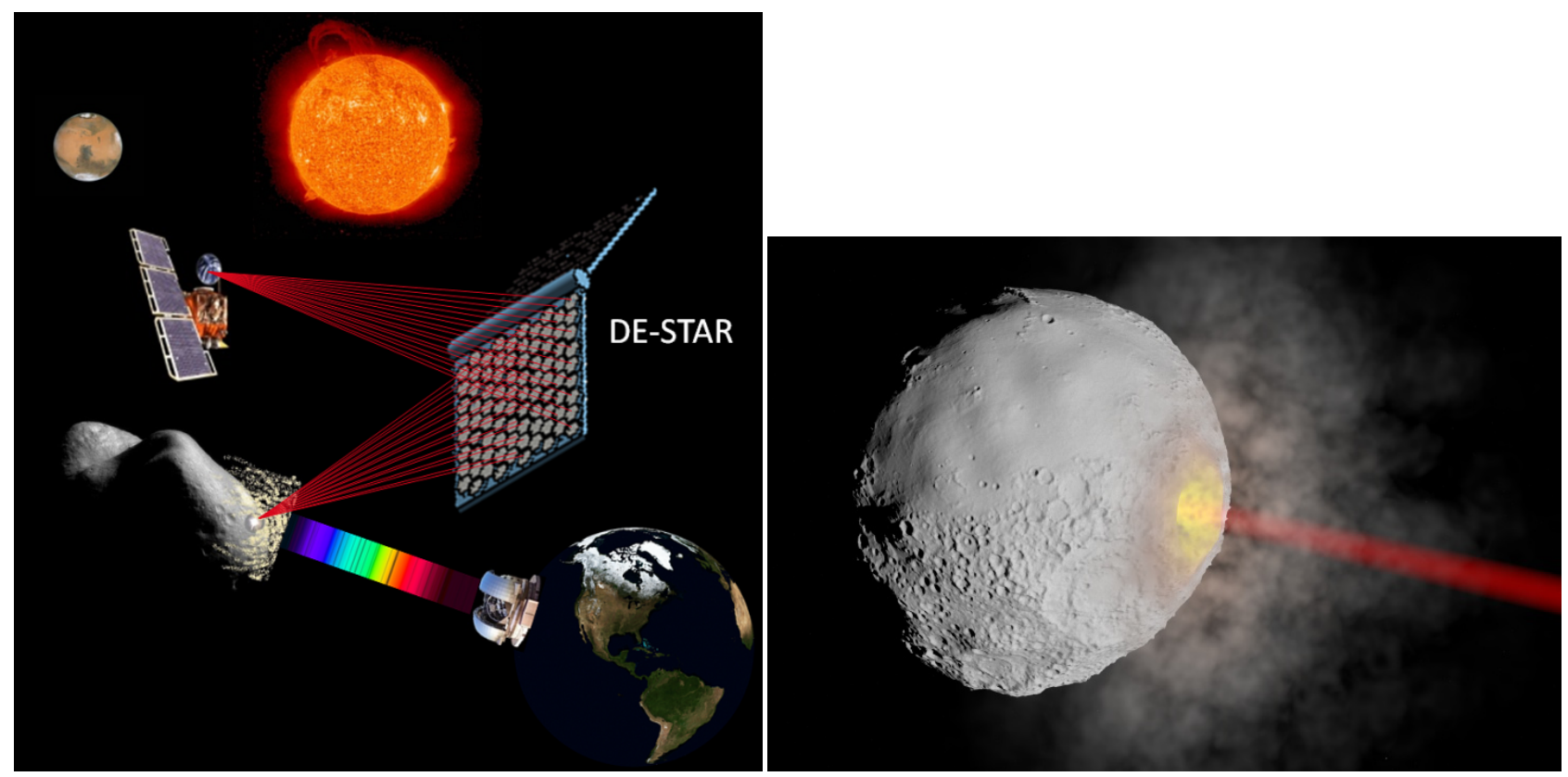

Figure 1.Left: Concept diagram of an orbiting DE-STAR engaged in multiple tasks including asteroid diversion, composition analysis and long range spacecraft power and propulsion. The system consists of an array of phase-locked lasers. By controlling the relative phases of individual laser elements, the combined beam can be directed to a distant target. Lasers are powered by a solar panel of effectively the same area as the laser array. A DE-STAR of sufficient size would be capable of vaporizing elements on the surface of asteroids. Given sufficient time, a threatening asteroid could be vaporized, deflected or disintegrated prior to impacting Earth. The ability to direct energy onto a distant target renders DE-STAR capable of many functions. Asteroid interrogation may be possible by viewing absorption lines as the heated spot is viewed through the ejected vapor plume. Photon pressure can be used to accelerate (and decelerate) interplanetary spacecraft, among many other possibilities. Right: Visualization with relevant physical phenomenon included at a flux of about $10 \mathrm{MW} /{ }^{2}$. Compare this to the picture of the laboratory test in Figure 13 where the bright high temperature spot is also visible with about the same flux. The plume density is exaggerated to show ejecta. Asteroid diameter is about that of Apophis $(325 \mathrm{~m})$ relative to the laser beam diameter $(30 \mathrm{~m})$. Target is at $1 \mathrm{AU}$.

\section{LASER PHASED ARRAYS}

\subsection{System architecture}

Planar arrays of phase-locked lasers have been developed in the laboratory. [Voronstov et al., 2009] describe a phased array of densely packed fiber laser collimators. The system utilizes adaptive dynamic phase distortion compensation to accomplish phase locking across the laser array. Other schemes for combining coherent beams have also been described [Fan, 2005]. The efficiency of laser fiber amplifiers has undergone a remarkable revolution in the last decade resulting from both the telecom industry and the commercial need for high power solid state lasers for machining among other tasks. With efficiencies already close to $50 \%$ for the lasers and with solar cells near $50 \%$ efficient we can realistically consider such a system. Our basic approach is to use existing technology without requiring any "miracles" but with reasonable expectations for modest improvements with an eye towards new devices that may be superior but the basic fact remains - it is now possible with high efficiency to convert light from the sun into a highly focused coherent beam capable of planetary scale defense. We feel it is now inevitable that this will be done and rapid progress with modest costs can begin this process that will lead to a full defensive capability. With efficiencies approaching unity we only project modest improvements (factor of 2 ) in efficiency but see a rapid improvement in power density $(\mathrm{kW} / \mathrm{kg})$. While current power density is about $0.2 \mathrm{~kW} / \mathrm{kg}$ using Ytterbium $(\mathrm{Yb})$ doped fiber amplifiers a relatively rapid roadmap to $1 \mathrm{~kW} / \mathrm{kg}$ is already in place. In the next decade we expect an order of magnitude increase in this. The current DARPA Excalibur program is one example of pursuing high efficiency fiber fed lasers. Excalibur goals are multi-kW fiber $1.06 \mu \mathrm{m}$ wavelength laser amplifiers with a target of $>0.2 \mathrm{~kW} / \mathrm{kg}$ with near $40 \%$ efficiency for the laser amplifier. Efficiency goals are comparable to current LED's that are already about 50\% efficient. Coincidentally, 
on the space PV side, the power density is nearly identical at $0.1 \mathrm{~kW} / \mathrm{kg}$ (ATK UltraFlex) with modest term possibilities for increasing this to $1 \mathrm{~kW} / \mathrm{kg}$. Recent work on Inverted Metamorphic Multijunction (IMM) cells promise $>0.5 \mathrm{~kW} / \mathrm{kg}$.

Long coherence length is critical and the existing fiber based laser amplifiers are already good enough (depending on the mode they are operated in), though new advances are becoming available to allow the SBS (Stimulated Brillouin Scattering) limit to be extended with even longer coherence lengths. With the current technology a DE-STAR 2 program could be started leading to launch and possibly a DE-STAR 3. We advocate a conservative and logical approach of rapidly building smaller and much lower cost units (DE-STAR 0 and 1), testing on the ground and then as technology catches up and technological and system problems arise and are solved move to larger systems, eventually leading to orbital testing and scale up to the full defensive goal. The system is a not binary in that small systems have immediate applications (DE-STAR 1 space debris for example) as larger systems are being developed for comet and small asteroid protection (DE-STAR 2) leading eventually to a DE-STAR 3 or 4.

As a goal we studied the feasibility of a system possessing the capability to evaporate, prior to impact, asteroids in the size range $150 \mathrm{~m}$ to $1 \mathrm{~km}$, and with typical orbital closing speeds. These stated capabilities drive system requirements into the multi-km class array size for both the diffraction limit of the optics and the power required. As a specific example, we could seek to evaporate an Apophis-class asteroid (325m diameter) with a worst case assumption of complete chemical binding and less than 1 year to evaporate the entire boloid, with a desired interdiction starting at $1 \mathrm{AU}$. A $10 \mathrm{~km}$ DE-STAR system would be capable of meeting the stated goal as shown in the calculations presented below. It is also fortuitous that the same size system required to form a small spot on the distant asteroid from the diffraction limit, assuming a wavelength near $1 \mu \mathrm{m}$, is also about the same size as needed to power the laser amplifiers in order to raise the flux to the evaporation point from converting sunlight that falls on the DE-STAR into electricity. At the Earth's orbit the "solar constant" is about $1400 \mathrm{~W} / \mathrm{m}^{2}$ or $1.4(140) \mathrm{GW}$ of sunlight on a $1(10) \mathrm{km}$ sized solar array. This is sufficient to power the entire system and no additional power is needed. This also forms a very large potential for an SPS system to send excess power to the Earth. By utilizing a filled array of solar powered phase locked lasers, there is a near ideal convergence of size required to both power the system and to produce the diffraction limited beam needed to begin vaporization. Baseline calculations are developed using a $1.06 \mu \mathrm{m}$ wavelength, to produce sufficient flux at $1 \mathrm{AU}$ that will sustain evaporation, which requires greater than approximately $5 \mathrm{MW} / \mathrm{m}^{2}$ flux at target. As stated existing $\mathrm{Yb}$ laser fiber amplifiers at $1.06 \mu \mathrm{m}$ wavelength have efficiencies near $40 \%$. Space solar PV has efficiency of about $35 \%$ in one sun (not concentrated) with near 50\% when concentrated. We assume modest efficiency improvements of both laser and $\mathrm{PV}$ to $70 \%$ which is not unreasonable in the realistic time scale of a full DE-STAR 4 system. We thus assume overall conversion efficiency of sunlight to laser power of about $50 \%$, resulting in approximately $0.7 \mathrm{GW} / \mathrm{km}^{2}$ of laser power. For a $1 \mathrm{~km}$ system laser power would be $0.7 \mathrm{GW}$ while a $10 \mathrm{~km}$ system would have laser power of $70 \mathrm{GW}$, which is more than sufficient for meeting the stated goal of surface vaporization at $1 \mathrm{AU}$ of all known materials. One major advantage of a phased array is that multiple independent beams can be produced, so multiple targets or efforts can be simultaneously engaged. For reference, we note that $70 \mathrm{GW}$ is the equivalent of about 1.4MT (megatons TNT - 1MT $4.2 \times 10^{15} \mathrm{~J}$ ) per day or about 500MT per year of potentially deliverable energy, a significant portion of the total currently active US nuclear arsenal. Note that in the process we also have 100GW of electrical energy produced or the equivalent of about 100 large utility nuclear reactors. This would allow a very large SPS if needed.

For DE-STAR, launch mass is critical in the costing analysis, so while the required efficiency is already effectively available, the power mass density is where we need to increase significantly. Solar PV cells can be extremely thin and low areal mass through focusing with thin film mirrors on solar PV may allow the lowest densities. For example, if $10 \mu \mathrm{m}$ thick PV could be produced (this is more of a mechanical issue as thinner films already exist on plastic) a $10^{4} \mathrm{~m}$ PV array would have a mass of about $3 \times 10^{6} \mathrm{~kg}$. The current issue for many space solar cells is the charged particle degradation which s currently met with a "cover glass" on each size of about $100 \mu \mathrm{m}$. If we could meet a laser power density of $10 \mathrm{~kW} / \mathrm{kg}$ (50x higher than current), then $70 \mathrm{GW}$ of fiber lasers would be $7 \times 10^{6} \mathrm{~kg}$. This mass does not represent the entire DE-STAR system, but the scale is not outrageous. $10 \mathrm{~kW} / \mathbf{k g}$ for laser mass density over 20 years is a goal but even the existing $0.2 \mathrm{~kW} / \mathrm{kg}$ density allows up to nearly a DE-STAR 3 using existing launcher capability. For reference, the International Space Station (ISS) mass is about $0.5 \times 10^{6} \mathrm{~kg}$ with much more than this being lifted into orbit as much of it was also returned in Shuttle missions. Conservatively we could thus say we already know how to launch few x $10^{6} \mathrm{~kg}$ class space mission as we already did so with the ISS. Either heavy lift chemical launchers would be needed to loft DE-STAR 4 modules, or a bootstrap ground based DE-STAR driven hybrid booster would be required. The modules are being designed around the existing heavy lift fairing size allowing for a 3-4 meter diameter class 
module. The modules can be quite thin and stacked during launch and assembled in orbit. Since the system is a phased array the structure does not need the structural integrity of a conventional mirror but rather must be stiff enough to have vibration modes that are below the metrology servo loop bandwidth as phase control is not handled by keeping the structure stiff but rather by measuring the relative position of each element adjusting the phase shifter in each amplifier to keep the beam on the target.

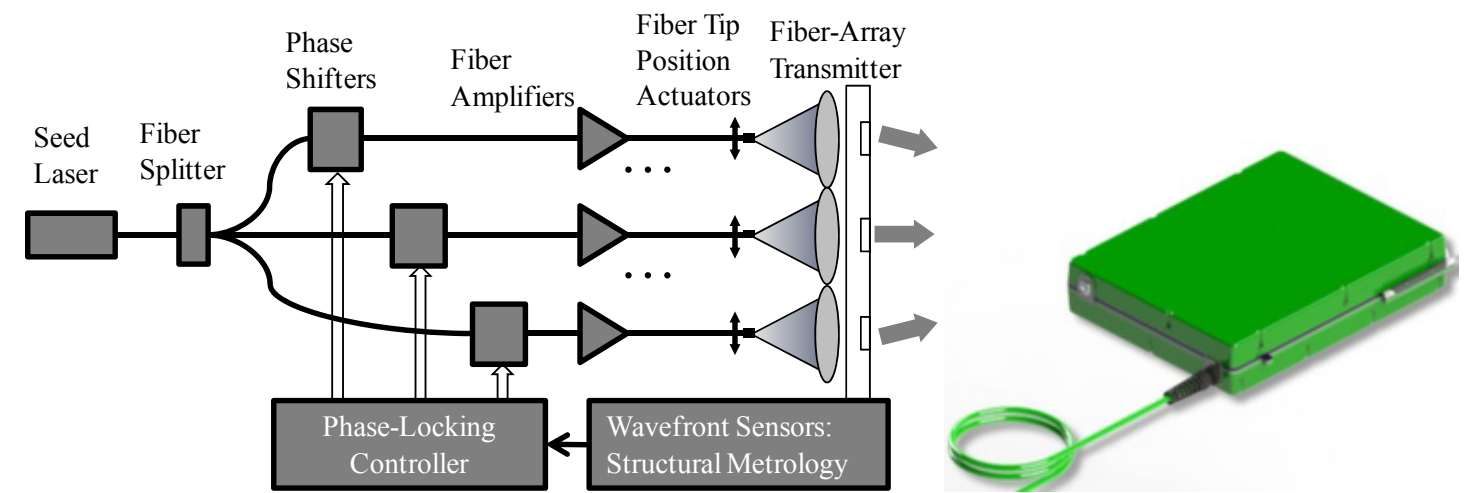

Figure1- Left: A system block diagram of the fiber amplifier configuration, based on work by [Voronstov et al., 2009]. Individual beams combine near the target. Here, coarse beam orientation is accomplished by moving individual fiber amplifier tips in relation to the transmitting element. Fine beam steering and beam combination at the target is accomplished by phase control. Right: Existing $1.5 \mathrm{~kW}$ Yb doped fiber amplifier of the type we baseline. Size is about 30 $\mathrm{x} 40 \mathrm{x} 10 \mathrm{~cm}$. We only need one of these per $2 \mathrm{~m}^{2}$ of the system.

While the baseline design is run in a continuous fashion ( $\mathrm{CW}$ mode) it is also possible to run the system pulsed if needed though short pulses are more problematic to phase properly. We have done extensive simulations and some laboratory testing shown below that indicate the debris field caused by the mass ejection should not significantly interfere with the incoming laser as the ejecta density is quite low (maximum near the surface is estimated to be $10^{-2} \mathrm{~kg} / \mathrm{m}^{3}$ and rapidly falling off due to the near isotropic ejecta emission into the space vacuum. Typical molecular ejecta speeds are $1-2 \mathrm{~km} / \mathrm{s}$. A reality check is to watch our laboratory tests in one atmosphere at up to $40 \mathrm{MW} / \mathrm{m}^{2}$ or to view a video of a laser milling machine. Our system is much like a laser heated target in a semiconductor fabrication system.

Thermal Dissipation - The average thermal load (to dissipate) of the system (independent of size) is about $500 \mathrm{~W} / \mathrm{m}^{2}$ which is approximately that of a person (or the Earth). It is equivalent to a $300 \mathrm{~K}$ blackbody. The average thermal load is extremely low. The average laser power is also quite low, being about $700 \mathrm{~W} / \mathrm{m}^{2}$ which is less than the solar "constant" on the surface of the Earth which is about $1,000 \mathrm{~W} / \mathrm{m}^{2}$. You could literally walk in front of the system when operational and not be harmed (laser glasses are recommended however). 


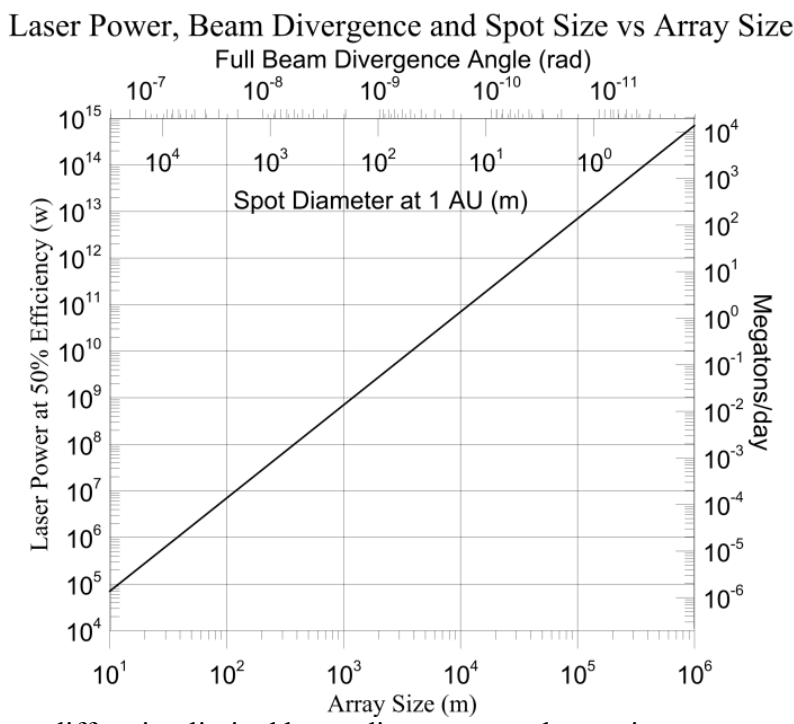

Figure 2 - DE-STAR laser power, diffraction limited beam divergence and spot size at target engagement of 1AU.

Optical Design - The optical design of a phased array is different than that of a classic optical telescope in that the phasing to achieve constructive interference (which is what allows the image to form) is not done with mechanical alignment as it is in a mirror or lens (where every part of the mirror is essentially a part of the overall "phased array") but rather the phasing is done by adjusting the phase at each sub element to achieve constructive interference at the target. We are an extremely narrow field of view system and thus we do not have many of the constraints of a classical optical system. We can be any shape for example. We are also extremely narrow bandwidth so thin film holographic grating diffractive "lenses" become viable. For simplicity we assume we will have a roughly planar design with each sub element being either a small reflector or possibly a thin film holographic lens. The latter has been tried in some narrow band receiving mode systems and extremely low areal densities have been achieved. This is an area we need further work to decide on the optimum approach. Our design is a large number of identical low power $\left(700 \mathrm{~W} / \mathrm{m}^{2}\right)$ modules that lend themselves to mass production. Ultra low mass holographic thin film large area "lenses" is particularly attractive but $\mathrm{SiC}$ or CFRP replicated reflective optics may be suitable with refinement to lower the mass. In our current baseline each element has a single fiber amplifier that feeds an optical element. A single $1 \mathrm{~kW}$ amplifier can feed a $1.5 \mathrm{~m}^{2}$ optic (mirror or lens). Coarse pointing could be accomplished using fiber tip position actuators behind the lens or mirror as appropriate. A fallback option would be to gimbal each element though this is more complex. Fine pointing is done with electronic phase adjusters at each amplifier input. The phase is also compared at the output and between elements. The metrology of the entire structure becomes a key part of the servo system. There have been a number of orbital programs looking at extremely high precision laser metrology over long baselines. The most extreme is the LISA gravitational wave detector that set a metric of $20 \mathrm{pm}$ resolution over $5 \times 10^{9} \mathrm{~m}$ baseline. This is vastly better than we require. We need about $0.1 \mu \mathrm{m}$ metrology $(\lambda / 10)$ over $10 \mathrm{~km}$ for the full DE-STAR 4. Similarly the AMD-MOST program has achieved 1 $\mathrm{nm}$ resolution over roughly $10 \mathrm{~m}$ baselines (limited by the vacuum chamber for testing). At longer wavelengths the Event Horizon telescope has phased locked $1.3 \mathrm{~mm}$ wavelength telescopes across the globe $\left(10^{7} \mathrm{~m}\right.$ baseline) and achieved $0.1 \mathrm{nrad}$ beam formation or the same as our goal. Radio Astron, a Russian and Earth long baseline interferometer has produced fringes corresponding to $0.04 \mathrm{nrad}$. Note that since the optical F\# is very large $\left(\sim 1.5 \times 10^{7}\right.$ for a DE-STAR 1AU target) since the asteroid is far awayand hence the beam is nearly parallel at the target with a large "depth of focus" $\sim \mathrm{F} \#^{2} \lambda \sim 2 \times 10^{8} \mathrm{~m}$. TheF\# (F number) is the ratio of $\mathrm{L} / \mathrm{d}$ where $\mathrm{L}$ is the target distance and $\mathrm{d}$ is the DE-STAR size.

There are a number of challenges to the optical design and the targeting servo system that need to be explored. Asteroids are dynamic and while motion in angle may be small to us it can still be significant. Typical asteroid move at $10-30 \mathrm{~km} / \mathrm{s}$ and with a $30 \mathrm{~m}$ beam this is $300-1,000$ beam diameters per second in the worst case. The system will be moving in its orbit, the Earth is moving ... There are a lot of issues to be worked out. The Hubble space telescope has about a $35 \mathrm{nrad}$ pointing stability over 24 hours as an example. We need better than $0.1 \mathrm{nrad}$ pointing ideally (our beam is $0.2 \mathrm{nrad}$ FWHM for a DE-STAR 4). Though, as we show below in the simulations, we have some latitude in this. We have started optical design and show a simple $2 \times 2$ array as an example using coherent beam combining in Zemax. 

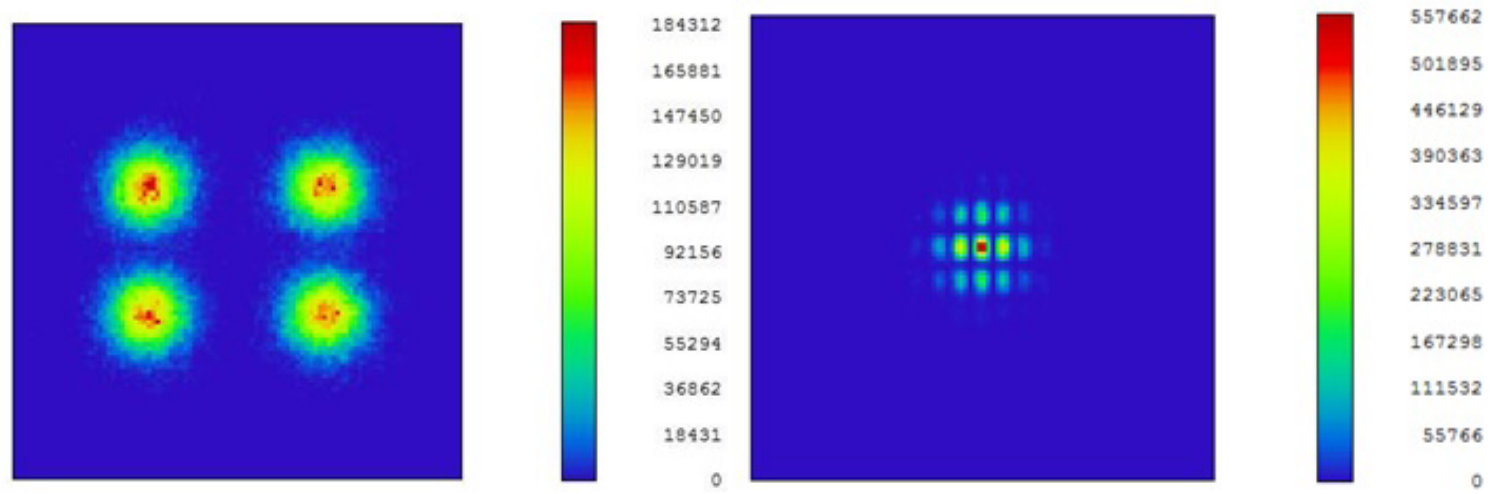

Figure 3 - Coherent beam combining of a 2x2 laser array in Zemax. The 4 individual elements are shown on the left and then when combined the central peak is intensified and the sidelobes suppressed. With additional elements the peak will grow and the sidelobes will decrease. Our baseline uses a filled close packed array to minimize sidelobes and to maximize the central peak.

Coherence Length requirements - For a phased array to work properly the light must be coherent over a time and thus length scale sufficient for all elements to be able to interfere. The coherence length required can be calculated by determining the length difference between the various elements with the most extreme case being the conservative limit. For a planar array of size $\mathrm{d}$ and a target of distance $\mathrm{L}$ away the path length difference between the central beam and the outermost beam is $\delta \sim \mathrm{d}^{2} / 8 \mathrm{~L}=\mathrm{d} / 8 \mathrm{~F} \#$ for the case of a target that is normal to the plane of the phased array. As we move off normal the path length difference is $\delta=1 / 2 \mathrm{~d} \sin (\theta)$ where $\theta$ is the angle of the target off the normal. The worst case is at right angles $(\theta=\pi / 2)$ where $\delta=\mathrm{d} / 2$. If there are controllable optical delays lines then these issues are drastically mitigated but it is preferable to have long coherence length so delays lines are needed. For a target at $\mathrm{L}=1 \mathrm{AU} \sim$ $1.5 \times 10^{11} \mathrm{~m}$ and a DE-STAR 4 with $\mathrm{d}=10^{4} \mathrm{~m}$ that $\mathrm{F} \# \sim 1.5 \times 10^{7}->\delta \sim 80 \mu \mathrm{m}$ corresponding to a coherence time $\mathrm{t}_{\mathrm{c}}=\delta / \mathrm{c} \sim$ 0.3 ps. For the worst case of $\delta=\mathrm{d} / 2$ the equivalent $\mathrm{t}_{\mathrm{c}}=\delta / \mathrm{c} \sim 17 \mu \mathrm{s}$. We want the laser coherence time to be greater than these times. The "coherence bandwidth" of the current $\mathrm{Yb}$ fiber amplifiers is intrinsically about 5-10 $\mathrm{KHz}$ (with corresponding coherence times $\mathrm{t}_{\mathrm{c}} \sim 100 \mu \mathrm{s}$ or comfortably longer than our worst case. For amplifiers run at their highest power level this "coherence bandwidth" is generally artificially broadened to about $10 \mathrm{GHz}(100 \mathrm{ps})$ in order to overcome what is known as the SBS (Stimulated Brillouin Scattering) limit that limits the amplification power. This is well above the normal incidence case but allows extremely little pointing margin. For example, even a 1 degree pointing difference will give a path length difference of $\delta=1 / 2 \mathrm{~d} \sin (\theta) \sim 90 \mathrm{~m}$ with a corresponding coherence time $\mathrm{t}_{\mathrm{c}}=\delta / \mathrm{c} \sim$ $300 \mathrm{~ns}$. When the amplifier is run at a few hundred watts vs kilowatts the "coherence bandwidth" is about $5-10 \mathrm{KHz}$ or less as above. The solution to this is to run at normal incidence (not really a good option), add path delay lines (also not a good option in general) or run the amplifiers well outside the SBS limit where the coherence time is longer. The latter is the preferred option. There is technology that has been developed that appears to allow the $\mathrm{Yb}$ amplifiers to run at both relatively high power and with long coherence time. This is one of the development items on the roadmap. Since volume (as opposed to mass) is not as much of an issue there may be a trade space that we can exploit to allow for better performance. Note that the deviation of the planar array from a sphere with radius $\mathrm{R}=\mathrm{L}$ is $\xi=\mathrm{d}^{2} / 8 \mathrm{R}=\mathrm{d}^{2} / 8 \mathrm{~L} \sim 80 \mu \mathrm{m}$ and deviation of the array plane from a classic optic with focal length $\mathrm{f}=\mathrm{L}$ is $\xi=\mathrm{d}^{2} / 16 \mathrm{f}=\mathrm{d}^{2} / 16 \mathrm{~L} \sim 40 \mu \mathrm{m}$. The array is indeed quite planar!

Space Qualification Issues -The DE-STAR system is a complex system of both power conversion (solar to electrical to laser) and metrology and targeting among many others. Solar PV is a mature technology and the space qualification and "rad hardening" issues are understood. The situation for fiber amplifiers needs to be addressed as a part of the roadmap. Much of this can be done on the ground in accelerator beam lines and some early long term space exposure will help with determining what issues, if any, are critical to address in this area. The long term exposure to radiation is not well understood for fiber amplifiers and needs to be addressed. Rad hardening of thin film holographic lenses (if we go this route) also needs to be addressed as does lowering the areal mass of space PV which is often dominated by the glass used to reduce charged particle (mostly electron) damage. 


\subsection{System requirements to evaporate asteroids}

We can calculate the energy required to melt and vaporize the various materials that are common in S-Type (Si rich), CType (Carbon rich) and M-Type (metal rich) asteroids. Comets are much easier to vaporize in that they do not require a high temperature to begin significant mass ejection. The gravitation binding energy of a molecule to a typical asteroid is very small and is negligible compared to the chemical binding energy. The chemical bonding energy that requires us to heat the spot to high temperature can be expressed through the heat of vaporization. The heat of fusion (melting) is a small fraction of the heat of vaporization. We have modeled the thermal interaction between the laser and asteroid in three ways. The first is a simple analysis based on power only with a flux equivalent to about a $6,000 \mathrm{~K}$ blackbody. The second method uses detailed calculations of the vapor pressure vs. temperature for every element and many of the estimated compounds that are thought to make up asteroids. This is a quasi $2 \mathrm{D}$ analysis in that includes radiation emission and mass ejection but ignores thermal conduction. The third method uses all the calculations from the second method but uses a full 3D FEA analysis of spherical (we can do any shape) asteroids with various thermal conductivities. All three methods give essentially the same answers but we wanted to confirm our calculations with increasingly sophisticated simulations. The final method is a laboratory test system that uses a 19 element laser array to produce a spot flux similar to that of the full DE-STAR 4 at $1 \mathrm{AU}$, namely about $40 \mathrm{MW} / \mathrm{m}^{2}$ and targets "rock" samples with similar compositions to asteroids. This testing has begun and will continue over the next year to cross check our simulations for evaporation rates, mass ejection densities and plume thrusts among other parameters. As expected when we exceed about $2 \mathrm{MW} / \mathrm{m}^{2}$ most materials begin to significantly vaporize.

The energy required to melt an asteroid is given by the heat of fusion and required increase in temperature to bring it to the melting point from (assumed) initial low temperature starting point. In practice this is small compared to the heat of fusion and heat of vaporization. The typical energy per $\mathrm{m}^{3}$ is of order $10^{10} \mathrm{~J}$ to vaporize most materials. This can be seen in the figures below where we model the vapor pressure in Pascals $\left(\mathrm{N} / \mathrm{m}^{2}\right)$ vs. T and vs. target flux for 93 elements. In addition we show models for 4 common asteroid molecular compounds. Even vapor pressures of $10^{3} \mathrm{~Pa}(0.01$ atmospheres) correspond to enormous reaction forces on the asteroid and large mass ejection rates. While we do not expect to see an asteroid of solid Tungsten we could mitigate it. Contrary to the small Iron rich meteorites that are found on the ground, a more typical asteroid looks more like the lunar surface and has quite low thermal conductivity and is thought to be a "rubble" pile in many cases, particularly for larger ( $>$ few hundred meters) asteroids. We have assumed the worst case of complete chemical binding (i.e. solid). In many cases asteroids will have significant low temperature volatile materials that may make mitigation much easier. Asteroids are also molecular rather than atomic in species in general but the conclusion are the same, namely at temperatures around $2,000-3,000 \mathrm{~K}$ or target fluxes of $10^{6}-10^{8} \mathrm{wW} / \mathrm{m}^{2}$ all known materials will undergo vigorous evaporation. What is critical is to increase the spot flux to the point where evaporation becomes large. It is not sufficient to simply apply a large amount of total power, there has to bea large flux to initiate evaporation.
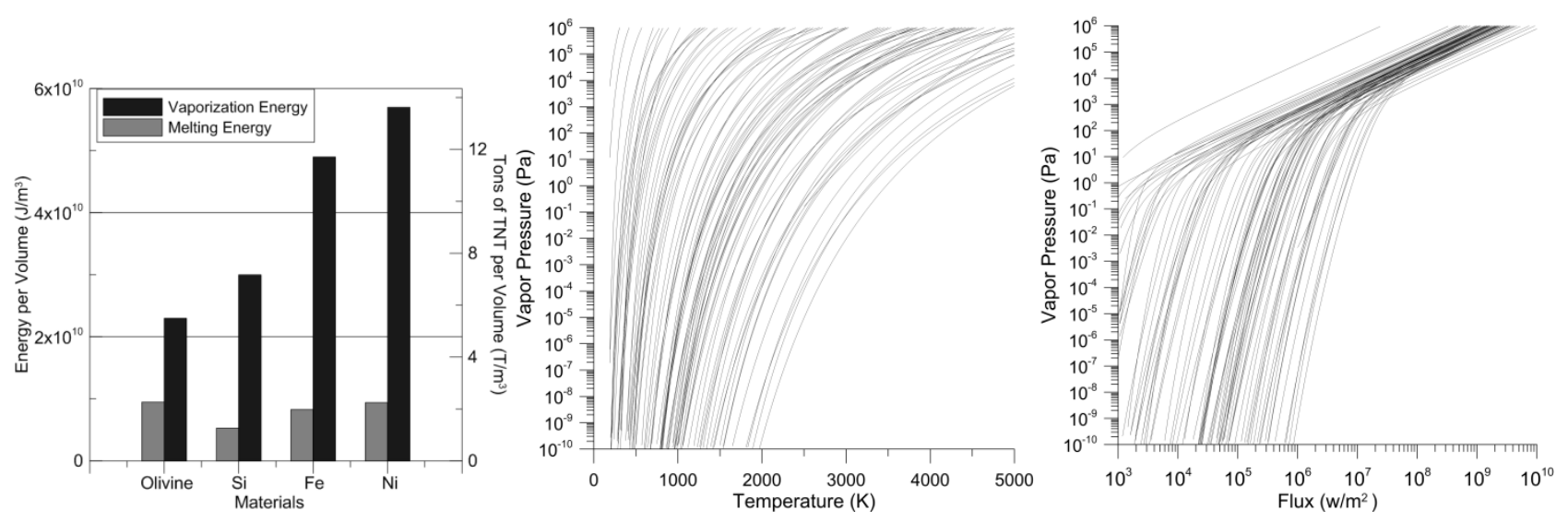

Figure 4 - Left: Melting and Vaporization energy per unit volume for S type (Si rich) asteroids. Middle: Vapor pressure vs. T for virtually all elements on the periodic table (93 are modeled). Right: Vapor pressure vs. target flux for the same 93 elements. The upper outlier is Mercury. 

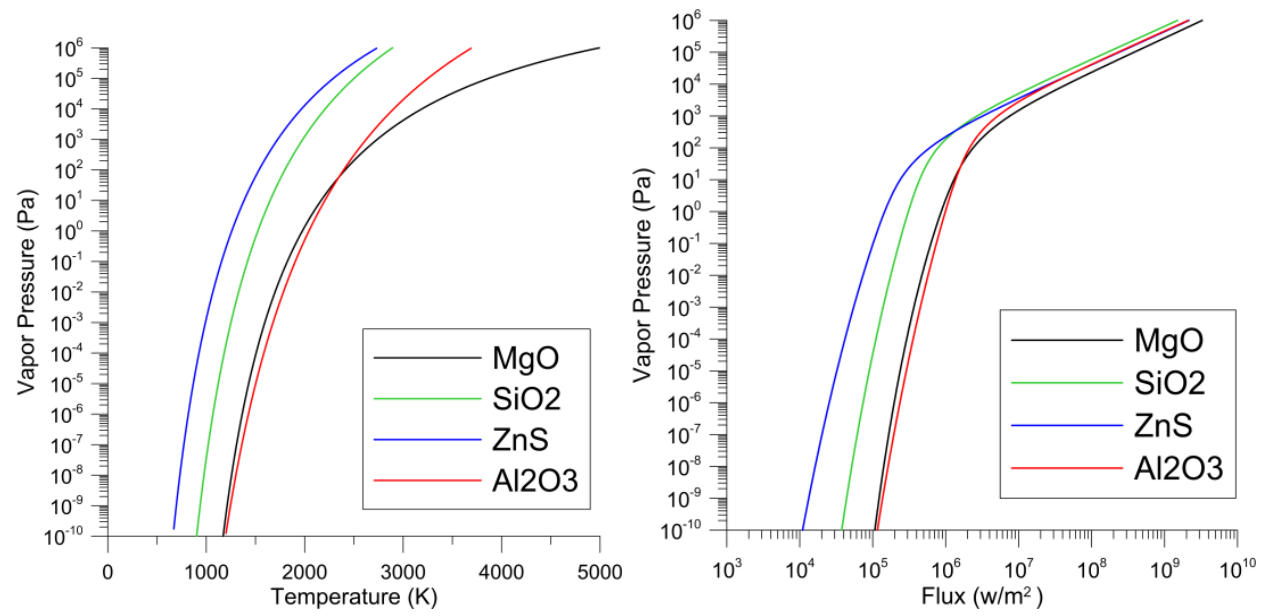

Figure 5 - Left: Vapor pressure vs. T for four common high temperature asteroid compounds. Right: Vapor pressure vs. target flux for the same found compounds. Note that at temperatures of $2,000-3,000 \mathrm{~K}$ or fluxes of about $10 \mathrm{MW} / \mathrm{m}^{2}$ the vapor pressure and hence mass ejection rates are very high.

Once we understand the material properties of the targets [Binzel et al 2009] we can design a system that is capable of evaporating them and in this process we divert them due to the large plume thrust generated. We see in the figures below at what distances we can begin to engage targets of differing compositions. For example a comet will begin evaporation at much lower flux than a rocky asteroid and thus we can begin to engage them at much lower total power levels and hence smaller systems or at much larger distances. These simulations assume the sun is also illuminating the targets which accounts for the lower temperature limit. This is a approximate as it is depends on the target reflectivity and orbit. The sun does not have a significant effect except in the case of comets. 


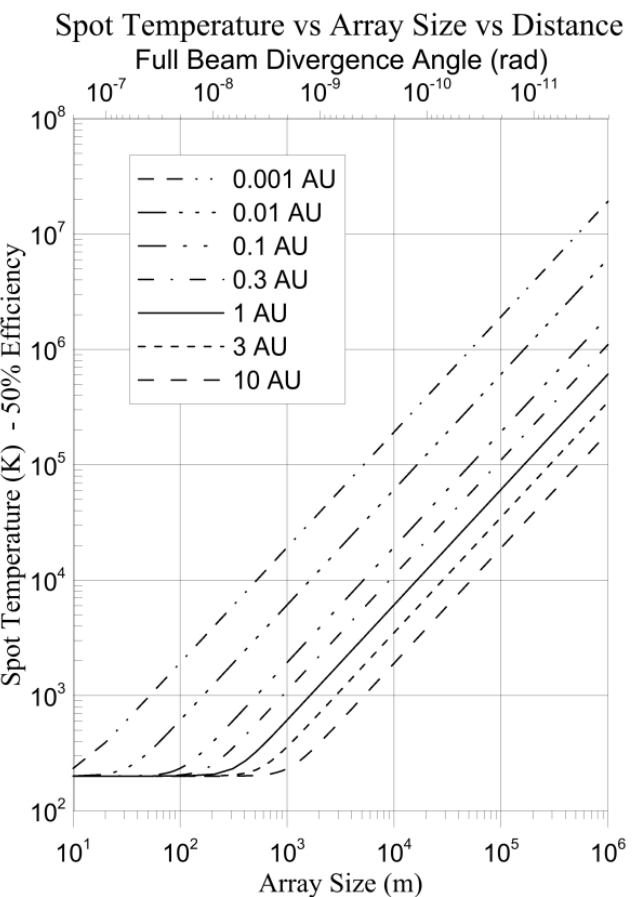

Evaporation Distance vs Array Size vs Evaporation Temperature

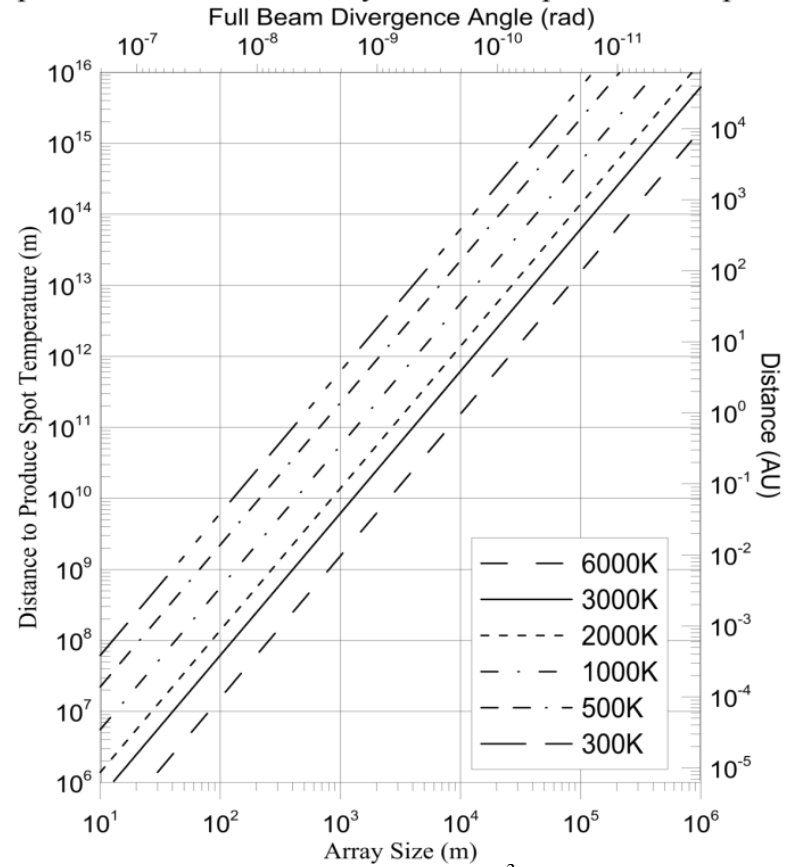

Figure 6 - Left: Spot temperature vs. DE-STAR array size for various target distances from $10^{-3}$ to 10 AU, including average solar illumination on asteroid (sets lower limit on asteroid or comet temperature). Right: Distance to target vs. array size for various spot temperatures from $300-6,000 \mathrm{~K}$. At $300 \mathrm{~K}$ icy comets become targets while at $6,000 \mathrm{~K}$ (hotter than sun) no known material survives.

\subsection{Detailed Thermal modeling}

Thermal modeling is critical. We take three approaches and all yield consistent results.

The basic equations are derived from energy conservation.

Power in (laser) $=$ Power out (radiation + mass ejection) $+\frac{d U}{d t}$ where $\mathrm{U}=$ Asteroid internal energy and $\frac{d U}{d t}$ is effectively from conduction. In the steady state $\frac{d U}{d t}=0$

$P_{\text {in }}=P_{\text {out }}+\frac{d U}{d t}$ with $\mathrm{U}=\int \rho c_{v} d v$ where $\quad c_{v}=$ specific heat $(\mathrm{J} / \mathrm{kg}-\mathrm{K})$

$$
\begin{array}{ll}
F_{L}=\text { Laser Flux }- \text { in } & \mathrm{W} / \mathrm{m}^{2} \\
F_{\text {rad }}=\text { Radiation Flux-out } & \mathrm{W} / \mathrm{m}^{2} \\
F_{\text {ejecta }}=\text { Ejecta Flux -out } & \mathrm{W} / \mathrm{m}^{2} \\
F_{\text {cond }}=\text { Thermal Conduction-in } & \mathrm{W} / \mathrm{m}^{2}
\end{array}
$$

$P_{\text {in }}=P_{\text {rad }}+P_{\text {Ejecta }}+P_{\text {cond }}$

$\oint\left(\bar{F}_{L}-\bar{F}_{\text {rad }}-\bar{F}_{\text {Ejecta }}-\bar{F}_{\text {cond }}\right) \cdot \hat{n} d A=0$

$=\int \nabla \cdot\left(\bar{F}_{L}-\bar{F}_{\text {rad }}-\bar{F}_{\text {Ejecta }}-\bar{F}_{\text {cond }}\right) d v=0$

Locally: 


$$
\begin{aligned}
& \bar{F}_{L}=\bar{F}_{\text {rad }}+\bar{F}_{\text {Ejecta }}+\bar{F}_{\text {cond }} \\
& \bar{F}_{\text {rad }}=\sigma T^{4} \hat{n} \\
& \bar{F}_{\text {Ejecta }}=\Gamma e \mathrm{Hv} \hat{n}=M^{1 / 2}(2 \pi R T)^{-1 / 2} \alpha_{e} 10^{[A-B /(T+C)]} \mathrm{Hv} \hat{n} \\
& \left|\bar{F}_{\text {rad }}\right|=\sigma T^{4} \\
& \left|\bar{F}_{\text {cond }}\right|=K \nabla T \\
& \left|\bar{F}_{\text {Ejecta }}\right|=\Gamma e * H v
\end{aligned}
$$

where $\mathrm{k}$ is the thermal conductivity (which can be position and temperature dependent) and $\Gamma e$ is the mass ejection flux $\left(\mathrm{kg} / \mathrm{m}^{2}-\mathrm{s}\right)$ and $\mathrm{Hv}$ is the heat of vaporization $(\mathrm{J} / \mathrm{kg})$

$\Gamma e=\frac{M \alpha_{e}\left(P_{v}-\mathrm{P}_{h}\right)}{\sqrt{2 \pi M R T}}=M^{1 / 2}(2 \pi R T)^{-1 / 2} \alpha_{e}\left(P_{v}-\mathrm{P}_{h}\right)$

$\mathrm{M}=$ Molar mass $(\mathrm{kg} / \mathrm{mole})$

$P_{v}=$ Vapor pressure $(\mathrm{Pa})$

$\mathrm{P}_{h}=$ Ambient vapor pressure $=0$ in vacuum

$\alpha_{e}=$ coef. of evaporation $0 \leq \alpha \leq 1$

We model the vapor pressure for each element and compound using a semi analytic form known as Antoine coefficients. $\log \mathrm{Pv}=\mathrm{A}-\mathrm{B} /(\mathrm{T}+\mathrm{C})$ where $\mathrm{A}, \mathrm{B}$ and $\mathrm{C}$ are unique per element and compound. These form the basis for Figures 3 and 4.

Hence:

$\mathrm{Pv}=10^{[A-B /(T+C)]}$
$\left|\bar{F}_{\text {Ejecta }}\right|=M^{1 / 2}(2 \pi R T)^{-1 / 2} \alpha_{e} 10^{[A-B /(T+C)]} H v$

We also assume a Gaussian profile for the laser as an approximation .

For Gaussian laser of power $P_{T}$

$\left|\bar{F}_{L}\right|=\frac{P_{T}}{2 \pi \sigma^{2}} e^{-r^{3} / 2 r^{2}}$

$\mathrm{r}=$ distance from spot center. In the approximation where the spot is small compared to the asteroid we have:

$\bar{F}_{L}=\frac{-P_{T}}{2 \pi \sigma^{2}} e^{-r^{3} / 2 r^{2}} \hat{n}$

In the dynamic case we also solve for transient heat flow by solving:

$$
\begin{aligned}
& \nabla \cdot(K \nabla T)+\frac{d}{d T}\left(\rho c_{v} T\right)=0 \\
& K \nabla^{2} T+\rho c_{v} \frac{d \mathrm{~T}}{d t}=0
\end{aligned}
$$

In the last equation we have assume $\mathrm{K}$ (thermal conductivity) is independent of position and $\rho, c_{v}$ are time independent. In the full 3D time dependent solution we use all of the above and simultaneously solve the equations using a $3 \mathrm{D}$ numeric solver (COMSOL in this case). 
In the 2D steady state solutions we assume the thermal conductivity is small (this is shown in our 3D simulations to be a valid assumption as well as from first principle calculations) and use a combination of radiation and mass ejection (phase change):

$$
\begin{aligned}
& \left|\bar{F}_{L}\right|=\left|\bar{F}_{\text {rad }}\right|+\left|\bar{F}_{\text {Ejecta }}\right|=F_{T} \\
& F_{T}=\sigma T^{4}+M^{1 / 2}(2 \pi R T)^{1 / 2} 10^{[A-B /(T+C)]} H v
\end{aligned}
$$

Inversion is not analytically tractable so we use numerical inversion to get $\mathrm{T}\left(\mathrm{F}_{\mathrm{T}}\right)$ which gives $P_{v}\left(F_{T}\right), \Gamma e\left(F_{T}\right)$ etc.

In this inversion we fit (to 10 th order typically) $\mathrm{T}=\sum_{n=1}^{N} a_{n}\left(\log F_{T}\right)^{n}$

We use the Gaussian approximation to the laser profile (this is not critical) to get $T(r), P_{v}(r), \Gamma_{e}(r)$ where $r$ is the distance from the center of the spot.

Since radiation goes as the $4^{\text {th }}$ power of $\mathrm{T}$ while the mass ejection from evaporation goes roughly exponentially in $\mathrm{T}$, at low flux levels the outward flow is completely dominated by radiation (you heat the asteroid slightly and it radiates). As the spot flux level increases (spot size shrinks or power increases or both) evaporation becomes increasingly dominant and eventually at about $\mathrm{T} \sim 2,000-3,000 \mathrm{~K}$ or fluxes of $10^{6}-10^{7} \mathrm{~W} / \mathrm{m}^{2}$ mass ejection by evaporation becomes the dominant outward power flow and (just as water boiling on your stove) the temperature stabilizes and increasing flux only increases the rate of mass ejection with only very small increases in temperature. To help understand this we plot the relationship between flux and temperature in the purely radiation dominated mode in Figure 7.

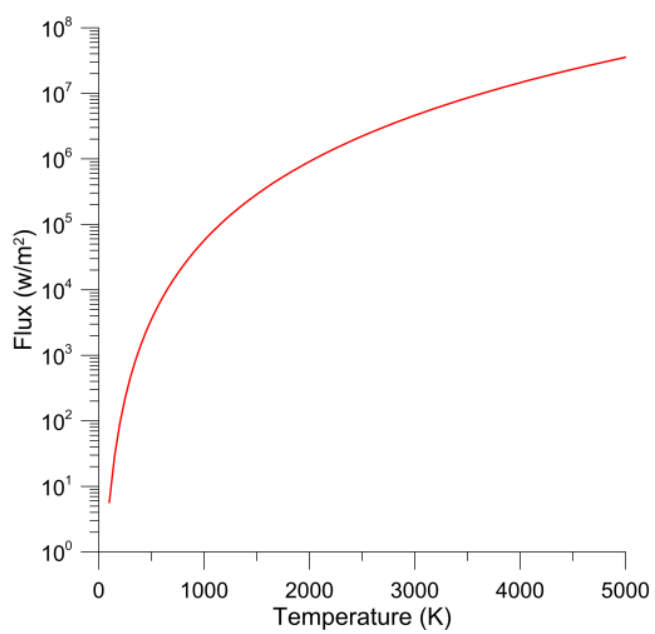

Figure 7- Relationship between flux and temperature in spot in the radiation dominated case. In reality the temperature rarely gets above $3,000 \mathrm{~K}$ as power in diverted from radiation to mass ejection.

\section{We will briefly summarize the results from the three methods below.}

- Energetics alone. Use heat of vaporization and set spot flux to $\mathrm{T} \sim 6,000 \mathrm{~K}$. No radiation or conduction included

- 2D - Model elements and compound vapor pressure vs. T. Include radiation emission. Ignore thermal conduction

- 3D - Full 3D FEA include phase change, vapor pressure, mass ejection, radiation and thermal conduction

1) Energetics alone - The heat of vaporization of a compound is the energy (per mole or per $\mathrm{kg}$ ) to remove it from the bulk. We can relate this to an effective speed and an effective temperature which are related to but somewhat different 
than the physical speed of ejection and the physical temperature of vaporization. To be more precise, the term evaporation refers to molecules or atoms escaping from the material (for example water evaporating) while boiling is the point at which the vapor pressure equals or exceeds the ambient pressure. At any non-zero temperature there is a probability of escape from the surface so evaporation happens at all temperatures and hence vapor pressure is a quantitative measure of the rate of evaporation. The heat of vaporization is also temperature and pressure dependent to some extent. As can be seen in the table below, the various materials that we plot vapor pressure vs. T and flux in Figure 4 above have relatively high effective temperatures reflecting the fact that there is a probability distribution of energies and that the increase in vapor pressure vs. $\mathrm{T}$ in Figure 4 shows that the thermal probability distribution has a "tail" allowing for escape from the surface at lower temperatures that one would naively conclude from a mean analysis only. A similar analogy is the Saha equation that relates the ionization fraction vs. temperature where a mean analysis would conclude that extremely high temperatures are required to ionize an atom but in fact significant ionization occurs at much lower temperatures due to the probability distribution tails. If we put power $\mathrm{P}_{\mathrm{T}}$ from the laser on the asteroid in a small enough spot to heat to above the radiation dominated point (typically 2,000-3,000K for "rocky" asteroids (vs 300-500K for comets)) we can compute the evaporation flux (mass ejection rate) as:

$\Gamma_{\mathrm{e}}=\mathbf{P}_{\mathrm{T}} / \mathbf{H v}$. This is the maximum possible rate of mass ejection. We will see we can get quite close to this maximum if we design the system properly.

Table 1 - List of thermophysical properties of common high temperature asteroid compounds.

\begin{tabular}{|l|l|l|l|l|l|}
\hline Material & $\mathrm{Hv}(\mathrm{kJ} / \mathrm{mole})$ & $\mathrm{M}(\mathrm{g} / \mathrm{mole})$ & $\mathrm{Hv}\left(10^{6} \mathrm{~J} / \mathrm{kg}\right)$ & $\mathrm{V}_{\text {eff }}(\mathrm{km} / \mathrm{s})$ & $\mathrm{T}_{\text {eff }}\left(10^{4} \mathrm{~K}\right)$ \\
\hline $\mathrm{SiO} 2$ & 143 & 60.1 & 2.38 & 1.54 & 0.573 \\
\hline $\mathrm{A} 2 \mathrm{O} 3$ & 293 & 102 & 2.87 & 1.69 & 1.15 \\
\hline $\mathrm{MgO}$ & 331 & 40.3 & 8.21 & 2.87 & 1.32 \\
\hline $\mathrm{ZnS}$ & 320 & 130 & 2.46 & 1.57 & 1.28 \\
\hline
\end{tabular}

Here $\mathrm{v}_{\text {eff }}=(\mathrm{Hv}(\mathrm{J} / \mathrm{kg}))^{1 / 2}$ and $\mathrm{T}_{\mathrm{eff}}=(\mathrm{M} * \mathrm{Hv}) / 3 \mathrm{R}$ where $\mathrm{R}=\mathrm{k}^{*} \mathrm{~N}_{\mathrm{A}} \sim 8.31$

\section{2) 2D thermal calculation}

As mentioned above in this calculation we will assume the thermal conduction is small compared to radiation and mass ejection (a good assumption for most asteroids). Using the equations above and the numerical inversions we can solve for the temperature distribution and thus the mass ejection and thrust on the asteroid among many other parameters. We summarize some of these in the figures below for $\mathrm{SiO}_{2}$. We allow $\sigma$ (sigma) in the Gaussian beam profile to vary to show the effects of non ideal beam formation as well as beam and pointing jitter. The diffraction limited $\sigma$ at $1 \mathrm{AU}$ should be about 5 meters. As can be seen we are quite tolerant to errors in beam formation, focus, beam bitter and pointing errors even beyond $10 \sigma$ as long as the power is high enough. The requirements on a low power system at equivalent distances are more severe. We also see that we come close to achieving the theoretical maximum mass ejection rate. Also, note the thrust (N) per watt is close to $0.001 \mathrm{~N} / \mathrm{W}$. This is comparable to the Shuttle SRB (solid Rocket Booster) in thrust per watt. This is not really surprising if you think of conventional propellants as being approximately thermal in nature with temperatures close to the maximum sustainable in the combustion chamber and exhaust nozzle (i.e. few $\times 10^{3} \mathrm{~K}$ ).

2a) Asteroid Plume thrust - The ejecta speed from the asteroid is also close to that of a conventional rocket (few $\mathrm{km} / \mathrm{s}$ ). The Shuttle SRB, for reference, has a power of about $13 \mathrm{GW}$ and a thrust of about $14 \mathrm{MN}$ and exhaust speed of around $2.6 \mathrm{~km} / \mathrm{s}$. Our computed thrust for a DE-STAR 4 with $70 \mathrm{GW}$ on target is about the same (thrust) as the SRB assuming our "exhaust nozzle" on the asteroid is nearly isotropic in the forward $2 \pi$. This "plume thrust" is what is responsible for the dramatic orbital diversion that is possible with the DE-STAR system. In a power limited system the thrust per watt is $1 / \mathrm{v}_{\text {rel }}$ where $\mathrm{v}_{\text {rel }}$ is the exhaust velocity. Thus a "photon rocket" or photon propelled system (one of the many other uses of the DE-STAR system is pushing a spacecraft via photon pressure) is the LEAST efficient method (in terms of thrust/watt) BUT in a mass limited system where mass is being ejected for propulsion (such as in a conventional rocket or an ion engine) the thrust to mass rate $(\mathrm{dm} / \mathrm{dt})$ is $\mathrm{V}_{\text {rel }}(=\mathrm{c}$ in relativistic limit)and hence photons are the MOST efficient (in terms of thrust/dm/dt). This is one of the basic rationales behind ion engines. They can achieve much higher (non thermal equilibrium) exhaust speeds (typically 10x or more) that a conventional propellant that is largely in thermal equilibrium. There have been proposals to use solar sails attached to asteroids as well as ion engines. Solar sails only have $\mathrm{F}$ (thrust) $=2 \mathrm{P} / \mathrm{c}$ where $\mathrm{P}$ is the power intercepted from the sun on the reflector. The factor of 2 is for perfect reflection. We will use this later for a DE-STAR standoff "photon rail gun" propulsion system [Bible et al., 2013]. The 
thrust per watt in this case is $\mathrm{F} / \mathrm{P}=2 / \mathrm{c} \sim 6.6 \mathrm{nN} / \mathrm{W}$ or more than $10^{5}$ times lower than our plume thrust. Current state of the art ion engines (eg. VASMIR VX-200) use $200 \mathrm{~kW}$ and produced $5.7 \mathrm{~N}$ with an exhaust speed of $50 \mathrm{~km} / \mathrm{s}(10 \mathrm{x}$ Shuttle main engine $\mathrm{H}_{2}-\mathrm{O}_{2}$ and 20x that of the SRB which is $\sim 2.6 \mathrm{~km} / \mathrm{s}$ ) and $72 \%$ efficiency using Argon and a plasma exhaust equivalent $\mathrm{T} \sim 10^{6} \mathrm{~K}$ with a thrust per watt of $2.85 \times 10^{-5} \mathrm{~N} / \mathrm{W}$ or about $3 \%$ of the SRB thrust/watt. This is fully consistent with the exhaust being about 20 times higher speed than the SRB and hence is should be 20 times less efficient $(5 \% \mathrm{x}$ 0.72 (eff) 3.6\%) in terms of thrust per watt. Of course, the major advantage of an ion engine compared to a conventional propellant is that it uses MUCH less propellant for an equivalent impulse (thrust * time), being about 20 times less and it can be throttled on and off easily. In the case of orbital modification of an asteroid we propose using the asteroid itself as the propellant and using a high power laser driven by solar PV if attachment to the asteroid is desired. This is a modified variant of the DE-STAR system. This is a much simpler and lower mass system compared to an ion engine (which is quite massive) with extremely long life. In theory the power required to get the same thrust as the VASMIR would be about 10-30 times less with this approach but this needs to be verified in lab testing, which we are starting on.

The plots in Figures 8 and 9 show the various parameters that come from the 2D analysis.

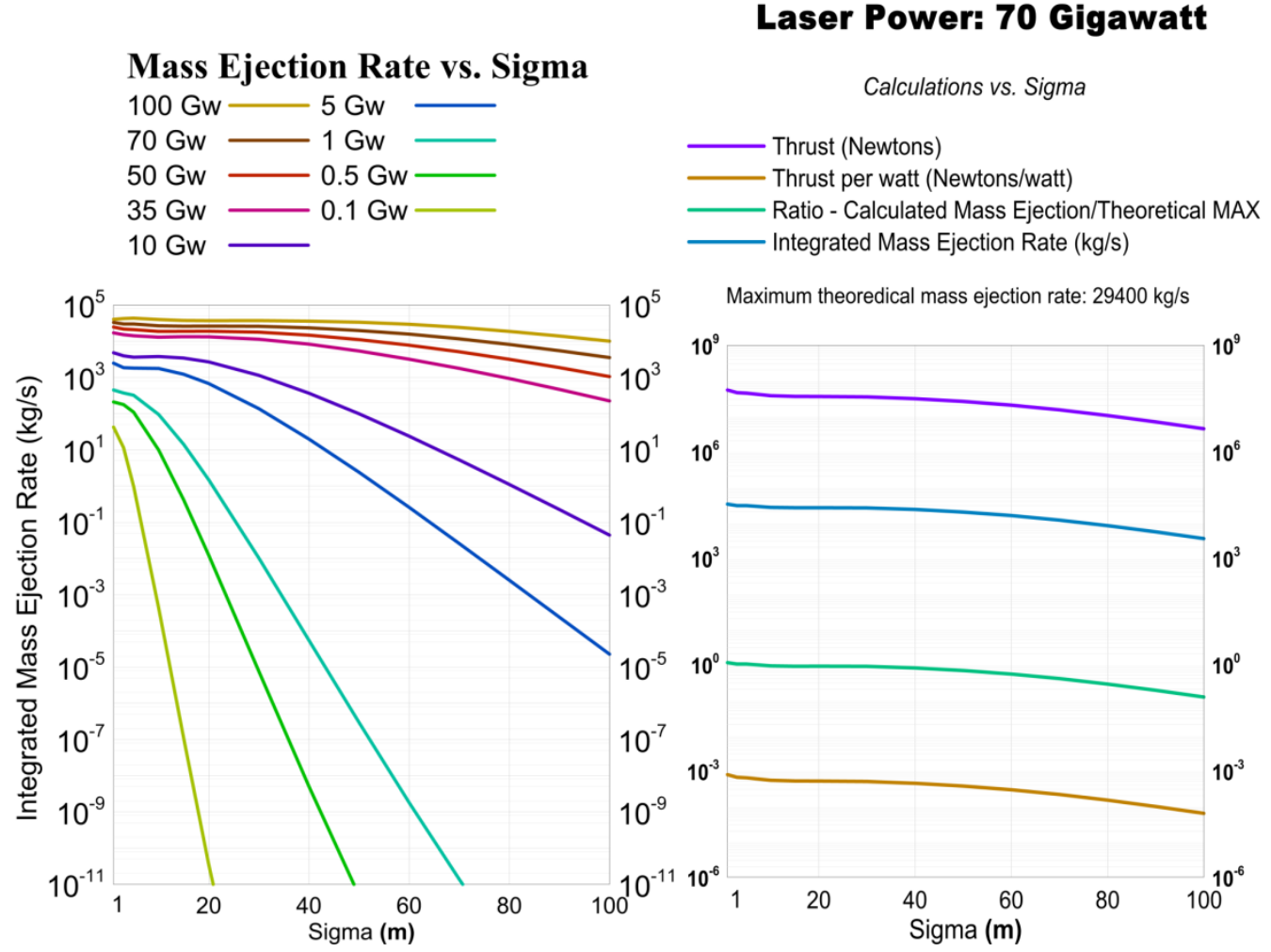

Figure 8 - Left: Mass ejection rate vs. sigma (in the assumed Gaussian laser beam profile) for various power levels for the compound $\mathrm{SiO}_{2}$. While this is done for a target at $1 \mathrm{AU}$ it is independent of distance. Note that at the higher power levels we are much more tolerant to errors that increase sigma. Right: Thrust, thrust per watt, ratio of integrated total mass ejection to maximum theoretical and integrated mass ejection vs. sigma for a DE-STAR with the target at 1AU. Nominal diffraction limited sigma is 5 meters but it is clear we have a very wide latitude (more than 10x) to absorb various errors that increase the effective sigma (beam formation, phase noise, beam jitter and pointing jitter.

Interaction simulations - In Figure 9 below we calculate various properties expected. This is done for $\mathrm{SiO}_{2}$ but the results are similar for the other compounds we have simulated. The vapor pressure and mass ejection and thrust have a roughly exponential rise with temperature but when computed vs. target flux they enter a nearly linear regime above about $10^{6}-10^{7} \mathrm{~W} / \mathrm{m}^{2}$. This is expected when the dominate flux is due to mass ejection and the vapor pressure, mass ejection rate and thrust are all approximately linear with power above this point. This is the point above which we want our flux to be in. The surface temperature does not change much in this regime just as a pot of boiling water remains at about $100 \mathrm{C}$ at sea level independent of how high you turn up the flame. This is the same linear regime. Notice the thrust starts at the photon thrust (absorbed in this case) of about $3.3 \mathrm{nN} / \mathrm{W}$ and rises more than 5 orders of magnitude to about 
$1 \mathrm{mN} / \mathrm{w}$ in the linear regime mentioned above. This value then essentially remains constant at high flux, until extremely high values are reached and ionization begins.

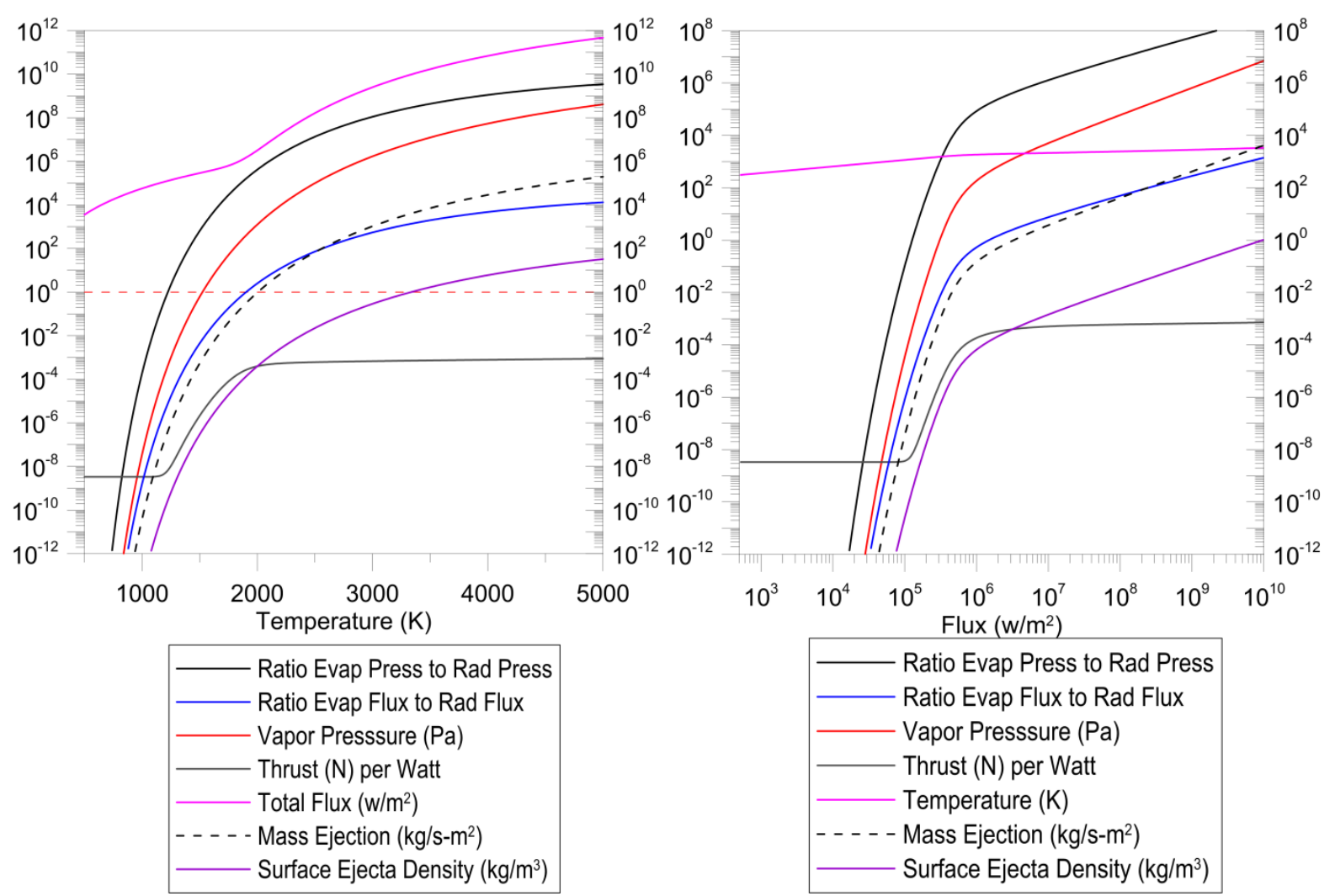

Figure 9 - Left: $\mathrm{Simulations} \mathrm{ofSiO}$ properties vs. temperature. Right: Simulations of $\mathrm{SiO}_{2}$ properties vs. flux.

\section{3) 3D Thermal calculations}

In the 3D simulations we use all of the above as shown in the thermal transport equations but we must numerically solve for the temperature distribution. In the model we put radiation, mass ejection and phase change and thermal conduction as well as solve for both the transient and steady state case. This was done with a 3D solver using COMSOL and modified to add mass ejection (phase change) for arbitrary materials.

3a) Thermal conduction - Unfortunately we cannot bring asteroids into the laboratory to study their thermal properties so we must rely on astronomical observations, primarily in the infrared to deduce their properties combined with assumptions about their formation and likely structure. [Mueller, 2007], [Mueller et al., 2007], [Harris, 1998] and [Delbo et al., 2007], among many others, have done excellent work in this area and we were able to use their results. One can derive the thermal properties by studying the time varying temperature as deduced from infrared observations. In this way the "thermal inertia $\Gamma\left(\mathbf{J} / \mathbf{m}^{2}-\mathbf{K}-\mathbf{s}^{1 / 2}\right)$ " and thermal conductivity $\mathrm{K}(\mathrm{W} / \mathrm{m}-\mathrm{K})$ are derived. The relationship between them is:

Thermal Intertia $(\Gamma)-\left(J / \mathbf{m}^{2}-K-\mathbf{s}^{1 / 2}\right)$ and Thermal conductivity: (K) - ( W/m-K)

$\Gamma=(\rho \mathrm{K} \mathrm{C})^{1 / 2}$

$\rho=$ density $\left(\mathrm{kg} / \mathrm{m}^{3}\right)$

$\mathrm{C}=$ heat capacity $(\mathrm{J} / \mathrm{kg}-\mathrm{K})$

hence:

$\mathrm{K}=\Gamma^{2} /(\rho \mathrm{C})$ 
The data is shown below (Figure 10) best fit to data from Delbo et al., 2007, where D is the asteroid diameter in $\mathrm{km}$ ) is:

$\Gamma=\mathrm{d}^{*} \mathrm{D}^{-\xi}$ with $\mathrm{d}=300(\mathrm{~km})$ and $\xi=0.4$

$\mathrm{K}=3 \times 10^{4} * \mathrm{D}^{-0.8} /(\rho \mathrm{C})$

The trend (with significant errors) is towards smaller asteroids having larger thermal conductivity and larger asteroids having smaller thermal conductivity. Some of this may be the "rock pile" effect for larger asteroids. It is the values that are of interest in our models. We have assumed a relatively conservative case of $K=1 \mathrm{~W} / \mathrm{m}-\mathrm{K}$.

To put this in perspective we use some values for common materials in the table below.

Table 2 - Common material thermal properties for comparison to the asteroid thermal properties in Figure 8.

\begin{tabular}{|l|l|l|l|l|}
\hline Material & $\mathbf{K}(\mathbf{W} / \mathbf{m}-\mathbf{K})$ & $\rho\left(\mathbf{k g} / \mathbf{m}^{\mathbf{3}}\right)$ & $\mathbf{C}(\mathbf{J} / \mathbf{k g}-\mathbf{K})$ & $\Gamma\left(\mathbf{J} / \mathbf{m}^{\mathbf{2}}-\mathbf{K}-\mathbf{s}^{\mathbf{1}}\right)$ \\
\hline Nickel & 91 & 8850 & 448 & $1.9 \times 10^{4}$ \\
\hline Iron & 81 & 7860 & 452 & $1.7 \times 10^{4}$ \\
\hline Granite & 2.9 & 2750 & 890 & 2600 \\
\hline Ice (solid) & 2.3 & 917 & 2000 & 2040 \\
\hline $\mathrm{SiO}_{2}$ (solid) & $1.04(200 \mathrm{C})$ & 2200 & 1000 & 1510 \\
\hline Water (liq 0 C) & 0.56 & 1000 & 4200 & 1500 \\
\hline Snow (firm) & 0.46 & 560 & 2100 & 740 \\
\hline Soil (sandy) & 0.27 & 1650 & 800 & 600 \\
\hline Pumice & 0.15 & 800 & 900 (varies significantly) & 330 \\
\hline Styrofoam & 0.03 & 50 & 1500 & 47 \\
\hline Air & 0.026 & 1.2 & 1000 & 5.6 \\
\hline Moon (regolith) & 0.0029 & 1400 & 640 & 51 \\
\hline
\end{tabular}

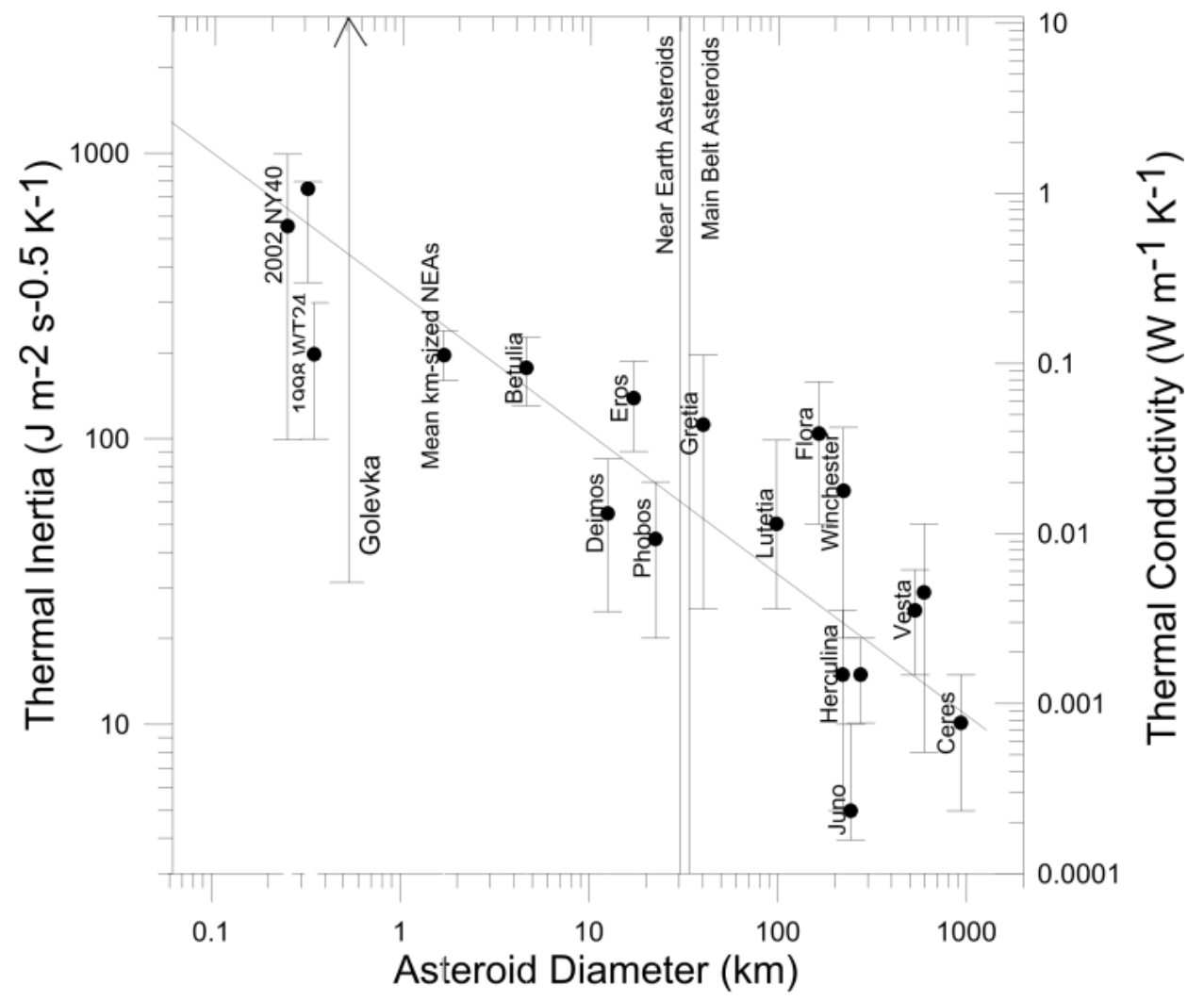

Figure 10 - Thermal properties measured for various asteroids from Delbo et al., 2007. 
3b) Rotating Asteroids - Asteroids do rotate but generally quite slowly. We do not have a complete picture of this but from the limited data on the rotation of larger bodies and the break up speed it is estimated that asteroids in the $0.1-1 \mathrm{~km}$ class typically rotate no faster than once per hour. As is seen in our transient thermal simulations below the mass ejection and hence thrust begins within about 1 second for a DE-STAR 4 at 1AU. It is largely a flux issue so that for the same flux at any distance the mass ejection remains at this rate. This is assuming solid $\mathrm{SiO}_{2}$ which is extremely conservative. We add loss to mimic the absorption qualities of asteroids which are very absorptive having typical reflection coefficients around $5 \%$. Thus a rotating asteroid with this rate $(1 \mathrm{hr})$ poses little problem. More interesting perhaps is can we spin up (or down) an asteroid depending on beam placement?

3c)3D results - We have run hundreds of 3D models and will show a few salient results. Perhaps the most interesting bottom line is that the simplest assumptions we started with, namely energetics only and conservation spot flux were borne out as being valid but we now have much more sophisticated tools with which to analyze and optimize the system.
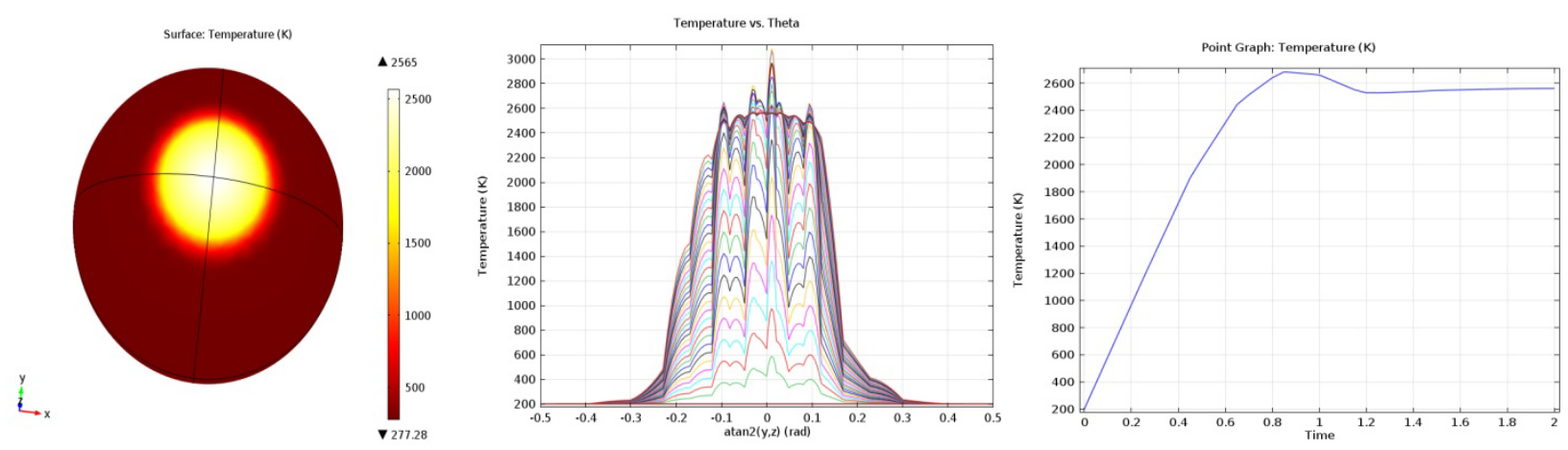

Figure 11 - All cases refer to $\mathrm{SiO}_{2}$ as the equivalent material. Left: Steady state surface temperature distribution for a 100 meter diameter asteroid at 1 AU with a DE-STAR 4 Gaussian beam de-rated to 50GW. Spot diameter is approximately 30 meter. Temperatures rise to the point of being mass mass ejection limited which is about, $2600 \mathrm{~K}$ in the center of the spot. Solar illumination with an isotropic average of $350 \mathrm{~W} / \mathrm{m}^{2}$ Middle: Temperature distribution vs. theta (angle from beam axis). High frequency sub structure is due to numerical meshing. Right: Transient time solution of temperature in the spot center (K) vs. Time (seconds) after the laser is turned on at $\mathrm{t}=0$. Initial temperature is $200 \mathrm{~K}$. Mass ejection begins within 1 second.

3d) Comparison of 2D and 3D simulations - While the 3D simulations give us time transient solutions and include full thermal conduction they lack the numerical flexibility of the $2 \mathrm{D}$ solutions. We compared the results of the temperature distributions for a Gaussian laser illumination and found them to be very close in their predictions. This gives us confidence that we can do both 2D and 3D simulations with high fidelity. The ultimate test will come when we compare the laboratory tests that we are embarking on and show some results below. In the figure blow we compare the temperature distribution for a 3D model (blue) with a 2D model (black). They have nearly identical results in the critical center of the spot and then differ in the wings. This is close enough for our needs now. As we refine the laboratory tests we will feed the results back into the models. 


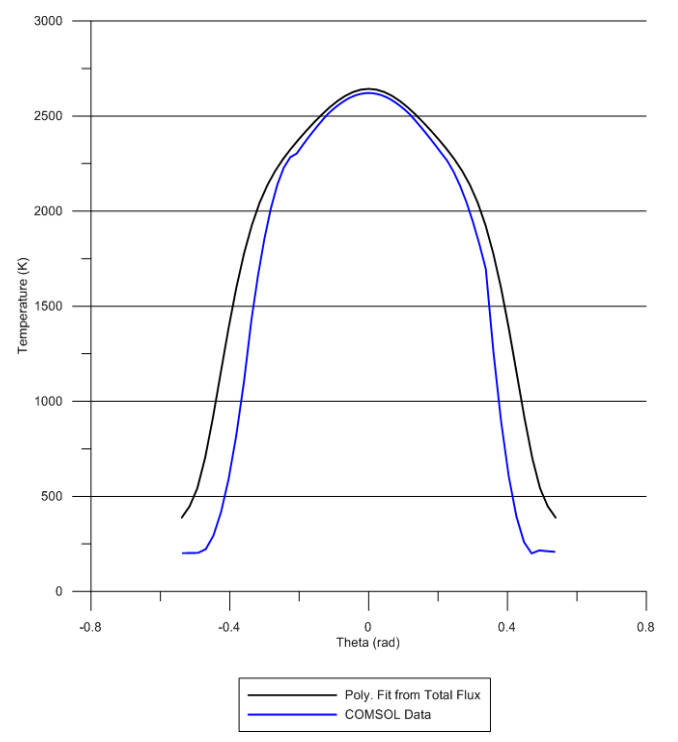

Figure 12 - Comparison of 2 and 3 D models temperature vs. theta (angle from beam axis on sphere) for $\mathrm{SiO}_{2}$ with $50 \mathrm{GW}$ total power and sigma $=5 \mathrm{~m}$ Gaussian beam illumination. Results are nearly identical in the critical central region.

\subsection{Orbital Diversion via Plume Thrust}

In general we do not need to evaporate the asteroid to avoid an impact scenario. It is sufficient to change its orbit enough to miss the earth. The ability to standoff and divert using the plume thrust that DE-STAR generates is an extremely attractive approach. Consider the example of Apophis. It is approximate $325 \mathrm{~m}$ in diameter with a mass of $4 \times 10^{10} \mathrm{~kg}$ and has an orbital speed of $30.7 \mathrm{~km} / \mathrm{s}$ with a 30 hour rotation. A direct hit would have a yield approaching 1 GT (Gigaton TNT). This would be a bad day. The momentum is approximately $\mathrm{p}=\mathrm{mv} \sim 1.2 \times 10^{15} \mathrm{~N}$-sec. If we could achieve our theoretical thrust to power ratio of $1 \mathrm{mN} / \mathrm{W}$ then the thrust with a DE-STAR 4 would be $7 \times 10^{7} \mathrm{~N}$. If we were to activate DE-STAR for one month we would achieve a change in momentum of Apohpis of $\delta p \sim 1.7 \times 10^{14} \mathrm{~N}$-s. The effect on the orbit depends on the details of when and where we begin the interaction but we can estimate the deflection angle to be $\delta \theta$ $\sim \delta \mathrm{v} / \mathrm{v}=\delta \mathrm{p} / \mathrm{p} \sim 0.14$ radians or a $\delta \mathrm{v} \sim 0.14 \mathrm{v} \sim 4.2 \mathrm{~km} / \mathrm{s}$. This is enormous by standards the deflection community speaks of. A simplistic distance deflection is given by $\delta \mathrm{r}$ (miss distance) $\sim \mathrm{L} \delta \theta\left(\mathrm{L}=1 \mathrm{AU} 1.5 \mathrm{x} 10^{11} \mathrm{~m}\right) \sim 2 \times 10^{10} \mathrm{~m} \sim 3,000 \mathrm{x}$ Earth radii. This is 50 times the Earth-Moon distance. This is obviously extremely conservative and we can back way off if needed.

\subsection{Laboratory testing}

A test system was constructed to check our calculations and simulations. This work is still ongoing but we show some of the results. The laser consisted of 19 fiber $\mathrm{CW}$ lasers each of which was homogenized in a 800 micron core fiber and then reimaged to simulate active phase control. Each fiber had a diameter of about 150 microns and was fed with 2.1 watt diode laser at $808 \mathrm{~nm}$. The beam diverge with a NA $\sim 0.2$ and re-converge with a roughly $1: 1$ ratio to produce a spot that was about $1 \mathrm{~mm}$ in diameter. Fluxes up to $40 \mathrm{MW} / \mathrm{m}^{2}$ are achieved which is close to the target of a DE-STAR 4 at $1 \mathrm{AU}$. For reference the surface of the sun (assuming a $5,800 \mathrm{~K}$ surface) has a flux of about $60 \mathrm{MW} / \mathrm{m}^{2}$. When we fire the laser at a target we do indeed create an extremely intense white hot spot that lights up the room and vaporizes every material we have tried. So far our tests are done outside the vacuum chamber but vac tests will begin shortly. Diagnostics include IR (out to 12 microns) and visible light cameras as well as a fiber fed optical spectrometer. Optical coupling from fiber tip to target was measured at about $90 \%$. Mass ejection was definitely observed (holes were punched through) but quantitative comparison to mass ejection model will be done in vacuum as the vapor pressure would have to exceed 1 atmosphere for normal evaporation. For Basalt the measured (in 1atm air) was $0.42 \mathrm{mg} / \mathrm{s}$ while the theoretical maximum for this test was $2.2 \mathrm{mg} / \mathrm{s}$. One significant issue is the complex nature of the test materials we are evaporating. We will use some standard targets in the vacuum tests. Air convection is also a serious issue so it is not surprising that our mass ejection is less than anticipated for a variety of reasons. We did try plain sand from the local beach and placed it in a small crucible and melted it into a glass ball is as well as vaporized some of it (Figure 14 Right). 


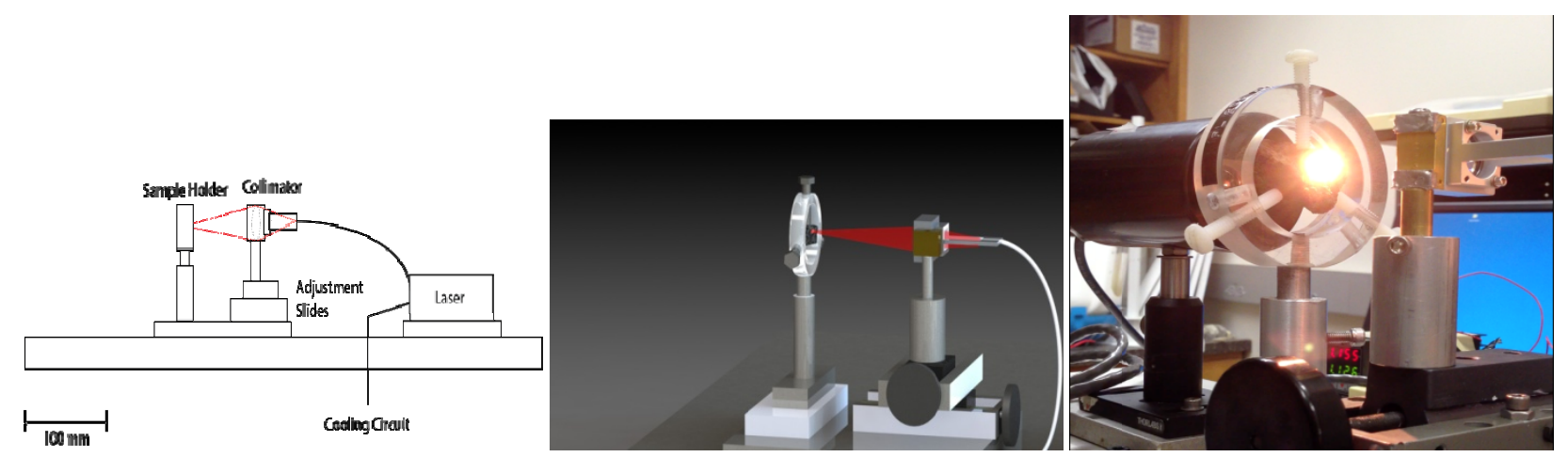

Figure 13 - Left: Cross section diagram showing laser (which is 19 individual fiber fed lasers) and the re-collimating optics. Middle: Rendering showing beam expansion and imaging as well as sample holder. Right: Laser firing at a target (basalt in this case).

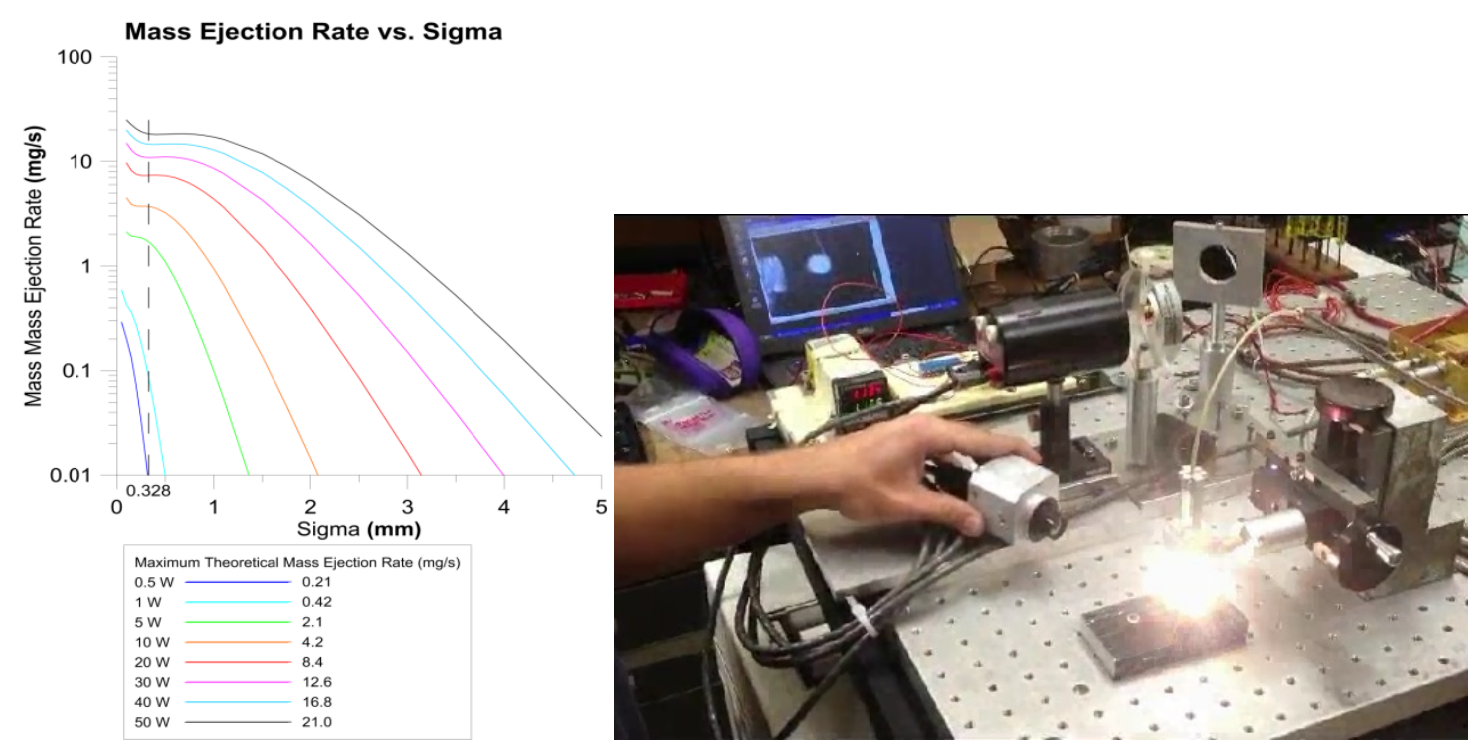

Figure 14 - Left: 2D simulation with laboratory test parameters. Similar to Figure 7 but set for lab testing. Plot is of expected mass ejection vs. sigma (Gaussian beam) for various power levels. Measured sigma based on hole size in targets is less than $330 \mu \mathrm{m}$. Sample is assumed to be $\mathrm{SiO}_{2}$. Right: Video 1:http://dx.doi.org/10.1117/12.2030228.1Picture of test system. Small camera is an 8-12 micron

FLIR IR microbolometer unit. Sample is sand.

\section{OTHER USES FOR DE-STAR}

\subsection{Summary of other uses}

DE-STAR is a standoff directed energy system and there a number of other uses that are possible. We have explored some in detail and are exploring others. Clearly if you can "laser machine" on solar system scales this brings up some thought provoking discussions.

Some of the more mundane ideas are:

- Space debris mitigation - a small unit (DE-STAR 1) is extremely effective against space debris. A unit attached to the ISS would be very useful in clearing out orbital debris.

- A LIDAR mode for refining the orbital parameters of asteroid. DE-STAR is extremely bright and makes an excellent "flashlight" to target asteroids with in order to detect and refine their positions. As an aid to existing efforts is can be quite useful The narrow bandwidth allow for extremely low background searches as well as Doppler velocity determination. 
- Standoff composition analysis - the bright heated spot might be used as a backlight to determine asteroid ejecta composition. We have begun an analysis of this to see what is feasible.

- Orbital capture - modifying the orbits of asteroids may allow for easier capture if desired.

- Beam power to distant probes - the system can be used to beam power to very distant spacecraft. At $1 \mathrm{AU}$ the flux is $70 \mathrm{MW} / \mathrm{m}^{2}$ or about 50,000 times the flux of the sun. At the edge of the solar system (30AU) it is about $80 \mathrm{~kW} / \mathrm{m}^{2}$. At $225 \mathrm{AU}$ the beam is about as bright as the sun is above the Earth atmosphere. Similarly it could be used to provide power to distant outposts on Mars or the Moon or literally to machine on the lunar surface (or possibly Mars). The latter would be a complex sociological and geopolitical discussion no doubt.

- Spacecraft rail gun mode - while photon pressure is modest it is constant until the beam diverges to be larger than the reflector. In a companion paper [Bible et al., 2013] discusses using this mode to propel spacecraft at mildly relativistic speeds. For example a $100 \mathrm{~kg}, 1,000 \mathrm{gk}, 10,000 \mathrm{~kg}$ spacecraft with a $30 \mathrm{~m}$ diameter $(9 \mathrm{~kg}-$ $10 \mu \mathrm{m}$ thick multi layer dielectric) reflector will reach $1 \mathrm{AU}$ ( Mars) in 3,10,30 days. Stopping is an issue! The $100 \mathrm{~kg}$ craft will be going at $0.4 \% \mathrm{c}$ at a $1 \mathrm{AU}$ and $0.6 \%$ at the edge of the solar system. This is $1,800 \mathrm{~km} / \mathrm{s}$ at the edge of the solar system with just a $30 \mathrm{~m}$ reflector. This speed is far greater than the galactic escape speed and nearly 100 times faster than the Voyager spacecraft. If a reflector could be built to intercept the beam out to the edge of the solar system (900 meter diameter) the same craft would be going $2 \%$ at the edge of the solar system and 3\% if illumination stayed on for about 2 months. We do not currently know how to build km class reflectors that are low enough mass though we do know how to build 30m reflectors and 100 meter appears feasible. There is work on Graphene sheets that may allow for future extremely large, extremely low mass reflectors that may allow for fully relativistic speeds. Future generation may build even larger DE-STAR 5 and 6 units to allow highly relativistic probes.

- Laser driven launch and boosters - a high power ground based DE-STAR could be used for launch purposes when used as an ablation [Campbell et al., 2003] or plume thrust driver. Similarly for orbital boost from LEO to GEO and beyond a DE-STAR could be extremely useful.

- SPS mode - beam power to the ground via microwave or $\mathrm{mm}$ wave. The system would produce about 100 GWe. US consumption is about $440 \mathrm{GWe}$ average $(1,400 \mathrm{~W} /$ person - ave).

- Interstellar beacon - we appear brighter than the brightest nighttime star at 1,000ly (typ distance to Kepler discovered exoplanets). Optical SEI use is being explored for both transmit and receive modes.

- Ultra high speed IR communications - the calculated data rates for DE-STAR to long range, even interstellar probes is enormous with $\mathrm{Mb} / \mathrm{s}$ speeds back to Earth from probes at the nearest stars for relatively small spacecraft transmitters and reflectors.

\section{CONCLUSIONS}

The DE-STAR system represents a solution to asteroids and comets that threaten the Earth. The same system can be used for a multitude of other purposes and thus is not a single use system waiting for an asteroid. Its use in spacecraft propulsion, space debris mitigation and SPS (Space Power Satellite) use could more than justify its cost let alone its ability to protect the Earth from catastrophe. Being modular and scalable the DE-STAR can be built in stages as technology progresses. Small DE-STAR $0(1 \mathrm{~m})$ and DE-STAR $1(10 \mathrm{~m})$ class units can be built, test and even flown on sub orbital platforms to test the basic concepts as small orbital version are built. The technology is improving rapidly and already nearly "there" in terms of conversion efficiency. There are many other uses that we have not touched on here for brevity. We propose a logical progression from the smaller DE-STAR ground and sub orbital units to small orbital units as the technology improves and laser mass power density improves until we can deploy a full scale system such as a DESTAR 4. As humanity becomes more technologically advanced even larger systems can be envisioned including systems that will allow the first interstellar probes. 


\section{ACKNOWLEDGEMENTS}

We acknowledge funding from the NASA California Space Grant NASA NNX10AT93H in support of this research. We are grateful to the Zemax support team for the optical simulations.

\section{REFERENCES}

[1] Bible, J., Johansson, I., Hughes, G. B., and Lubin, P. M., "Relativistic Propulsion Using Directed Energy,” Proc. Of SPIE, Optics \& Photonics (2013).

[2] Hughes, G. B., Lubin, P. M., Bible, J., Bublitz, J., Arriola, J., Motta, C., Suen, J., Johannson, I., Riley, J., Sarvian, N., Wu, J., Milich, A., and Pryor, M., "DE-STAR: Phased Array Laser Technology for Planetary Defense and Other Scientific Purposes," Proc. of SPIE, Optics \& Photonics (2013).

[3] Vorontsov, M. A., Weyrauch, T., Beresnev, L. A., Carhart, G. W., Liu, L., and Aschenback, K., "Adaptive Array of Phase-Locked Fiber Collimators: Analysis and Experimental Demonstration," IEEE Journal of Selected Topics in Quantum Electronics 15, 269 (2009).

[4] Fan, T. Y., "Laser Beam Combining for High-Power, High-Radiance Sources," IEEE Journal of Selected Topics in Quantum Electronics 11, 567 (2005).

[5] Binzel, R. P., Rivkin, A. S.,, Thomas, C. A., Vernazza, P., Burbine, T. H., DeMeo, F. E., Bus, S. J., Tokunaga, A. T., and Birlan, M., "Spectral Properties and Composition of Potentially Hazardous Asteroid (99942) Apophis," Icarus 200, 480-485 (2009).

[6] Mueller, M., "Surface Properties of Asteroids from Mid-Infrared Observations and Thermophysical Modeling," (2007).

[7] Mueller, M., Harris, A. W., Fitzsimmons, A., "Size, albedo, and taxonomic type of potential spacecraft target Asteroid (10302) 1989 ML,"

[8] Harris, A. W., "A Thermal Model for Near-Earth Asteroids," Icarus 131, 291-301 (1998).

[9] Delbò, M., Cellino, A., Tedesco, E. F., "Albedo and Size Determination of Potentially Hazardous Asteroids: (99942) Apophis," Icarus 188, 266-270 (2007).

[10] Campbell, J. W., Phipps, C., Smalley, L., Reilly, J., Boccio, D., "The Impact Imperative: Laser Ablation for Deflecting Asteroids, Meteoroids, and Comets from Impacting the Earth," AIP conference proceedings 664, 509520 (2003). 